\title{
Global Well-Posedness for Energy Critical Fourth-Order Schrödinger Equations in the Radial Case
}

\author{
Benoit Pausader \\ Communicated by Terence Tao, received May 31, 2007, revised June 18, $200 \%$.

\begin{abstract}
Energy-critical fourth order Schrödinger equations are investigated. We establish local well-posedness and stability in a general setting, and we prove global well-posedness and scattering in the defocusing case for radially symmetrical initial data.
\end{abstract}

\section{Contents}

1. Introduction 197

2. Notations 199

3. Strichartz-type Estimates 200

4. Local and Global Existence in the subcritical case 204

5. Local existence in the critical case 205

6. Stability in the critical case 208

7. Almost conservation Laws 211

8. Global Existence 215

9. Scattering for the critical equation $\quad 222$

$\begin{array}{ll}\text { References } & 224\end{array}$

\section{Introduction}

Fourth-order Schrödinger equations have been introduced by Karpman [13] and Karpman and Shagalov [14] to take into account the role of small fourth-order dispersion terms in the propagation of intense laser beams in a bulk medium with Kerr nonlinearity. Such fourth-order Schrödinger equations are written as

$$
i \partial_{t} u+\Delta^{2} u+\varepsilon \Delta u+f\left(|u|^{2}\right) u=0
$$

1991 Mathematics Subject Classification. 35, 42, 76.

Key words and phrases. Energy-critical fourth order Schrödinger equations, local wellposedness, global well-posedness. 
where $\varepsilon \in \mathbb{R}$ is essentially given by $\varepsilon= \pm 1$ or $\varepsilon=0$, and $u: I \times \mathbb{R}^{n} \rightarrow \mathbb{C}$ is a complexvalued function. Sharp dispersive estimates for the biharmonic Schrödinger operator in (1.1), namely for the linear group associated to $i \partial_{t}+\Delta^{2}+\varepsilon \Delta$, have recently been obtained in Ben-Artzi, Koch, and Saut [1], while specific nonlinear fourthorder Schrödinger equations as in (1.1) have been recently discussed in Fibich, Ilan, and Papanicolaou [5], Guo and Wang [9], Hao, Hsiao, and Wang [10, 11], and Segata [25]. Fibich, Ilan and Papanicolaou [5] describe various properties of the equation in the subcritical regime, with part of their analysis relying on very interesting numerical developments. Guo and Wang [9] prove global well-posedness and scattering in $H^{s}$ for small data. Hao, Hsiao and Wang $[\mathbf{1 0}, \mathbf{1 1}]$ discuss the Cauchy problem in a high-regularity setting. Segata $[\mathbf{2 5}]$ proves scattering in the case the space dimension is one. Related equations also appeared in Fibich, Ilan, and Schochet $[\mathbf{6}]$, Huo and Jia [12], and Segata $[\mathbf{2 3}, \mathbf{2 4}]$.

When $n \leq 4$, and $f$ has polynomial growth, equation (1.1) is subcritical and its analysis follows from standard developments. When $n \geq 5$ criticality in the energy space appears with the power-type nonlinearity $f(u)=|u|^{2^{\sharp}}-2 u$, where $2^{\sharp}=2 n /(n-4)$ is the critical exponent for the embedding of $H^{2}$ into Lebesgue's spaces. Following classical notations, we let $H^{2}$ be the space of square integrable functions whose first and second derivatives are also square integrable. We concentrate here on (1.1) with a pure power-type nonlinearity and aim in proving global existence in the critical defocusing regime of (1.1) for arbitrary initial data. Global well-posedness for the classical second order Schrödinger equation in the critical defocusing regime with a pure power-type nonlinearity has been recently established in a series of papers by Bourgain [2], Colliander, Keel, Staffilani, Takaoka, and Tao [4], Grillakis [8], Ryckman and Visan [22], Tao [26], and Visan [29]. We refer also to Kenig and Merle [17] for a similar result in the focusing case for solutions whose energy and kinetic energy are smaller than that of the ground state, and to Killip, Visan, and Zhang [18], where a quadratic potential is added to the classical second order Schrödinger equation. By analogy with second order Schrödinger equations it can be conjectured that global well-posedness holds true for (1.1) with a pure power-type nonlinearity, in the critical defocusing regime, for arbitrary initial data. We prove such global well-posedness when the initial data is radially symmetrical.

As already mentioned, the equations we consider in this paper correspond to (1.1) when $f$ is a pure power-type nonlinearity. They are written as

$$
i \partial_{t} u+\Delta^{2} u+\varepsilon \Delta u+\lambda|u|^{p-1} u=0,
$$

where $\lambda \in \mathbb{R}$, and $p \in\left(1,2^{\sharp}-1\right]$. The energy critical regime in (1.2) corresponds to the case $p=2^{\sharp}-1$, and the defocusing regime to the case $\lambda>0$. As a remark, when $\varepsilon=0,(1.2)$ enjoys scaling invariance. The scaling, as expressed in (4.1) below, preserves the homogeneous Sobolev space $\dot{H}^{2}$ when $p=2^{\sharp}-1$. Our main result states as follows.

Theorem 1.1. Let $n \geq 5, \varepsilon \in \mathbb{R}, \lambda>0$, and $p \in\left(1,2^{\sharp}-1\right]$. For any radially symmetrical data $u_{0} \in H^{2}$ there exists a unique global solution $u \in C\left(\mathbb{R}, H^{2}\right)$ of (1.2) such that $u(0)=u_{0}$.

When $p$ is subcritical, or $u_{0}$ has small energy and $p$ is critical, the radially symmetrical assumption on $u_{0}$ in Theorem 1.1 is not needed. We refer to Corollary 4.1 in Section 4 and Corollary 5.1 in Section 5 for more details on such assertions. 
We mainly concentrate in this paper on the critical case of (1.2). In this case we also prove stability for all $\varepsilon$, see Proposition 6.1 in Section 6 , and scattering when $\varepsilon \leq 0$, see Proposition 9.1 and Proposition 9.2 in Section 9. Needless to say, scattering and stability are important notions for physical considerations.

Our paper is organized as follows. We fix notations in Section 2. Strichartz type estimates, a classical one and one with gain of derivatives, relying on the dispersion estimates in Ben-Artzi, Koch, and Saut [1] , and on the Strichartz type estimates of Keel and Tao [16], are proved in Section 3. The local theory for (1.2), without the radially symmetrical assumption, and for arbitrary $\lambda$ 's, is established in Sections 4 to 6 . The subcritical case of (1.2) is briefly discussed in Section 4. Local existence in the critical case is proved in Section 5, and stability in the sense of Tao and Visan [28] is discussed in Section 6. We prove localized Morawetz estimates and almost local conservation of mass in Section 7 . Such estimates and conservations laws are crucial for the proof of Theorem 1.1. The theorem is proved in Section 8 following the strategy initiated in Bourgain $[\mathbf{2}]$ and developed in Tao [26]. Finally, in Section 9, we briefly discuss the scattering assertion we made after Theorem 1.1.

\section{Notations}

We fix notations we use throughout the paper. In what follows, we denote by $C$ a generic constant that is allowed to depend on the dimension and the nonlinearity through $|\lambda|$ and $p$. The exact value of that constant may change from line to line. We write $C(a), C(a, b)$ when there is more dependence. More significative constants are often denoted by $K, K_{1}, K_{2}$ to highlight their role. We let $L^{q}=L^{q}\left(\mathbb{R}^{n}\right)$ be the usual Lebesgue spaces, and $L^{r}\left(I, L^{q}\right)$ be the space of measurable functions from an interval $I \subset \mathbb{R}$ to $L^{q}$ whose $L^{r}\left(I, L^{q}\right)$ norm is finite, where

$$
\|u\|_{L^{r}\left(I, L^{q}\right)}=\left(\int_{I}\|u(t)\|_{L^{q}}^{r} d t\right)^{\frac{1}{r}}
$$

Two important conserved quantities of equation (1.1) are the mass and the energy defined by

$$
M(u)=\int_{\mathbb{R}^{n}}|u(x)|^{2} d x
$$

on what concerns the mass, and

$$
E(u)=\frac{1}{2} \int_{\mathbb{R}^{n}}\left(|\Delta u(x)|^{2}-\varepsilon|\nabla u(x)|^{2}+F\left(|u(x)|^{2}\right)\right) d x
$$

on what concerns the energy, where $F(s)=\int_{0}^{s} f(t) d t$. Several norms have to be considered in the analysis of the critical case of (1.2). For $I \subset \mathbb{R}$ an interval, they are defined as

$$
\begin{aligned}
& \|u\|_{M(I)}=\|\Delta u\|_{L^{\frac{2(n+4)}{n-4}\left(I, L^{\frac{2 n(n+4)}{n^{2}+16}}\right)},}, \\
& \|u\|_{W(I)}=\|\nabla u\|_{L} \frac{2(n+4)}{n-4}\left(I, L^{\left.\frac{2 n(n+4)}{n^{2}-2 n+8}\right)},\right. \\
& \|u\|_{Z(I)}=\|u\|_{L^{\frac{2(n+4)}{n-4}}\left(I, L^{\frac{2(n+4)}{n-4}}\right)} \text {, and } \\
& \|u\|_{N(I)}=\|\nabla u\|_{L^{2}\left(I, L^{\frac{2 n}{n+2}}\right)} .
\end{aligned}
$$


Accordingly, we let $M(\mathbb{R})$ be the completion of $C_{c}^{\infty}\left(\mathbb{R}^{n+1}\right)$ with the norm $\|\cdot\|_{M(\mathbb{R})}$, and $M(I)$ be the set consisting of the restrictions to $I$ of functions in $M(\mathbb{R})$. We adopt similar definitions for $W, Z$, and $N$.

An important quantity, which turns out to be closely related to the mass and the energy, is the functional $\mathcal{E}$ defined for $u \in H^{2}$ by

$$
\mathcal{E}(u)= \begin{cases}\int_{\mathbb{R}^{n}}|\Delta u(x)|^{2} d x & \text { if } \varepsilon=0 \\ \int_{\mathbb{R}^{n}}\left(|\Delta u(x)|^{2}-\varepsilon|\nabla u(x)|^{2}+|u(x)|^{2}\right) d x & \text { if } \varepsilon= \pm 1 .\end{cases}
$$

Note that when $\varepsilon=0, \mathcal{E}(u)$ is nothing but $\|u\|_{\dot{H}^{2}}^{2}$, while when $\varepsilon= \pm 1, \mathcal{E}(u)$ controls the full inhomogeneous norm $\|u\|_{H^{2}}=\|\Delta u\|_{L^{2}}+\|u\|_{L^{2}}$.

In what follows we let $\mathcal{F} f=\hat{f}$ be the Fourier transform of $f$ given by

$$
\hat{f}(\xi)=\frac{1}{(2 \pi)^{\frac{n}{2}}} \int_{\mathbb{R}^{n}} f(y) e^{i\langle y, \xi\rangle} d y
$$

for all $\xi \in \mathbb{R}^{n}$. The biharmonic Schrödinger semigroup is defined for any tempered distribution $g$ by

$$
e^{i t\left(\Delta^{2}+\varepsilon \Delta\right)} g=\mathcal{F}^{-1} e^{i t\left(|\xi|^{4}-\varepsilon|\xi|^{2}\right)} \mathcal{F} g .
$$

Let $\psi \in C_{c}^{\infty}\left(\mathbb{R}^{n}\right)$ be supported in the ball $B_{0}(2)$, and such that $\psi=1$ in $B_{0}(1)$. For any dyadic number $N=2^{k}, k \in \mathbb{Z}$, we define the Littlewood-Paley operators $P_{N}$ by

$$
\widehat{P_{N} f}(\xi)=(\psi(\xi / N)-\psi(2 \xi / N)) \hat{f}(\xi) .
$$

These operators commute one with another. They also commute with derivative operators and with the semigroup $e^{i t\left(\Delta^{2}+\varepsilon \Delta\right)}$. In addition they are self-adjoint and bounded on $L^{p}$ for all $1 \leq p \leq \infty$. Moreover, they enjoy the following Bernstein property:

$$
\left\||\nabla|^{ \pm s} P_{N} f\right\|_{L^{p}} \leq C N^{ \pm s}\left\|P_{N} f\right\|_{L^{p}} \leq C N^{ \pm s}\|f\|_{L^{p}},
$$

for all $s \geq 0$, and all $1 \leq p \leq \infty$, where $|\nabla|^{s}$ is the classical fractional differentiation operator, and $C>0$ is independent of $f, N$, and $p$. Given $a \geq 1$, we let $a^{\prime}$ be the conjugate of $a$, so that $\frac{1}{a}+\frac{1}{a^{\prime}}=1$.

\section{Strichartz-type Estimates}

We prove Strichartz type estimates for solutions of the linear equation associated with the biharmonic Schrödinger operator and forcing term $h \in L_{l o c}^{1}\left(I, H^{-4}\right)$ for $I \subset \mathbb{R}$ an interval. In other words, for

$$
u(t)=e^{i t\left(\Delta^{2}+\varepsilon \Delta\right)} u_{0}+i \int_{0}^{t} e^{i(t-s)\left(\Delta^{2}+\varepsilon \Delta\right)} h(s) d s,
$$

where $u_{0} \in L^{2}$. Key estimates in this section are given by the dispersion estimates of Ben-Artzi, Koch, and Saut [1] . Let $I_{\varepsilon}$ be given by

$$
I_{\varepsilon}(t, x)=\frac{1}{(2 \pi)^{n}} \int_{\mathbb{R}^{n}} e^{i t\left(|\xi|^{4}-\varepsilon|\xi|^{2}\right)-i\langle x, \xi\rangle} d \xi
$$

Using (2.5), one sees that $I_{\varepsilon}$ is the fundamental solution of (3.1). Let also $\alpha \in \mathbb{N}^{n}$. Then, according to Ben-Artzi, Koch, and Saut [1] , the following estimates hold true. Namely, 
(a) estimates for the homogeneous equation:

$$
\left|D^{\alpha} I_{0}(t, x)\right| \leq C t^{-\frac{n+|\alpha|}{4}}\left(1+t^{-\frac{1}{4}}|x|\right)^{\frac{|\alpha|-n}{3}}
$$

for all $t>0$ and all $x \in \mathbb{R}^{n}$,

(b) short time estimates for the inhomogeneous equation:

$$
\left|D^{\alpha} I_{\varepsilon}(t, x)\right| \leq C t^{-\frac{n+|\alpha|}{4}}\left(1+t^{-\frac{1}{4}}|x|\right)^{\frac{|\alpha|-n}{3}}
$$

for all $0<t \leq 1$ and all $x \in \mathbb{R}^{n}$, or all $t>0$ and all $|x| \geq t$,

(c) long time estimates for the inhomogeneous equation:

$$
\left|D^{\alpha} I_{-1}(t, x)\right| \leq C t^{-\frac{n+|\alpha|}{2}}\left(1+t^{-\frac{1}{2}}|x|\right)^{|\alpha|}
$$

for all $t \geq 1$ and all $|x| \leq t$,

where $D$ stands for differentiation in the $x$ variable. Useful consequences of (3.3)(3.5) are that

$$
\left|D^{\alpha} I_{\varepsilon}(t, x)\right| \leq C|t|^{-\frac{n}{2}}
$$

and that

$$
\left\|e^{i t\left(\Delta^{2}+\varepsilon \Delta\right)} g\right\|_{L^{p}} \leq C|t|^{-\frac{n}{4}\left(1-\frac{2}{p}\right)}\|g\|_{L^{p^{\prime}}}
$$

for all $\alpha$ such that $|\alpha|=n$, all $p \in[2, \infty]$, all $g \in L^{p^{\prime}}$, and all time $t \neq 0$, where $p^{\prime}$ is the conjugate exponent of $p$ and, if $\varepsilon=1$, we also require that $|t| \leq 1$. Inequality (3.6) is a direct consequence of (3.3)-(3.5). Inequality (3.7) follows from the remark that by (3.2)-(3.5) we can write that

$$
\left\|e^{i t\left(\Delta^{2}+\varepsilon \Delta\right)} g\right\|_{L^{\infty}}=\left\|I_{\varepsilon}(t) * g\right\|_{L^{\infty}} \leq\left\|I_{\varepsilon}\right\|_{L^{\infty}}\|g\|_{L^{1}} \leq C|t|^{-\frac{n}{4}}\|g\|_{L^{1}}
$$

while Plancherel's theorem ensures that $e^{i t\left(\Delta^{2}+\varepsilon \Delta\right)}$ is bounded $L^{2} \rightarrow L^{2}$. By the Riesz-Thorin theorem, interpolation between the $L^{2}$ and $L^{\infty}$ bounds gives (3.7).

Following standard notations, we say that a pair $(q, r)$ is Schrödinger admissible, for short S-admissible, if $2 \leq q, r \leq \infty,(q, r, n) \neq(2, \infty, 2)$, and

$$
\frac{2}{q}+\frac{n}{r}=\frac{n}{2} \text {. }
$$

Also we add the terminology that a pair $(q, r)$ is biharmonic admissible, for short $B$-admissible, if $2 \leq q, r \leq \infty,(q, r, n) \neq(2, \infty, 4)$, and

$$
\frac{4}{q}+\frac{n}{r}=\frac{n}{2} \text {. }
$$

Our Strichartz type estimates for (3.1) are stated as follows.

Proposition 3.1. Let $u \in C\left(I, H^{-4}\right)$ be a solution of $(3.1)$ with $\varepsilon \in\{-1,0,1\}$ on an interval $I=[0, T]$. If $\varepsilon=1$, suppose also $|I| \leq 1$. For any B-admissible pairs $(q, r)$ and $(\bar{q}, \bar{r})$

$$
\|u\|_{L^{q}\left(I, L^{r}\right)} \leq C\left(\left\|u_{0}\right\|_{L^{2}}+\|h\|_{L^{\bar{q}^{\prime}\left(I, L^{\bar{r}^{\prime}}\right)}}\right)
$$

whenever the right hand side in (3.10) is finite, where $C$ depends only on $n$, and $\bar{q}^{\prime}$ and $\bar{r}^{\prime}$ are the conjugate exponents of $\bar{q}$ and $\bar{r}$. Besides, for any $S$-admissible pairs $(q, r)$ and $(a, b)$, and any $s \geq 0$,

$$
\left\||\nabla|^{s} u\right\|_{L^{q}\left(I, L^{r}\right)} \leq C\left(\left\||\nabla|^{s-\frac{2}{q}} u_{0}\right\|_{L^{2}}+\left\||\nabla|^{s-\frac{2}{q}-\frac{2}{a}} h\right\|_{L^{a^{\prime}}\left(I, L^{b^{\prime}}\right)}\right)
$$


whenever the righthand side in (3.11) is finite, where $C$ depends only on $n$, and $a^{\prime}$ and $b^{\prime}$ are the conjugate exponents of $a$ and $b$.

It can be noted that, when $n \geq 4$, estimates (3.11) implies estimates (3.10).

Proof. Estimates (3.10) are easy to obtain. They directly follow from the linear estimates of Ben-Artzi, Koch and Saut (3.7) with $p=+\infty$ and $p=2$, and the theorem in Keel and Tao [16] applied to the operator $\mathcal{U}(t)$, where

$$
\mathcal{U}(t)= \begin{cases}e^{i t\left(\Delta^{2}+\varepsilon \Delta\right)} & \text { if } \varepsilon \leq 0 \\ e^{i t\left(\Delta^{2}+\Delta\right)} \mathbf{1}_{[-1,1]}(t) & \text { if } \varepsilon=1 .\end{cases}
$$

Now we turn to the proof of (3.11). Since the free propagator $e^{i t\left(\Delta^{2}+\varepsilon \Delta\right)}$ commutes with the derivative operator $|\nabla|^{s}$, it suffice to prove (3.11) with $s=0$. For $N$ a dyadic integer and $P_{N}$ as in (2.6), we let $Q_{N}=P_{N / 2}+P_{N}+P_{2 N}$. The LittlewoodPaley projector $Q_{N}$ is such that $P_{N} Q_{N}=P_{N}$. For any $\lambda>0$, let $d_{\lambda}$ be the rescaling operator defined on all functions $g$ by $d_{\lambda} g(x)=\lambda^{\frac{n}{2}} g(\lambda x)$. We consider the family of operators

$$
\mathcal{V}_{N}(t)=d_{N} Q_{N} \mathcal{U}(t)
$$

By Plancherel's theorem and since $d_{N}$ is an isometry on $L^{2}$, we get that

$$
\left\|\mathcal{V}_{N}(t)\right\|_{L^{2} \rightarrow L^{2}} \leq C \text {. }
$$

Independently, it is easily seen that

$$
Q_{N} e^{i t\left(\Delta^{2}+\varepsilon \Delta\right)} u_{0}=e^{i t\left(\Delta^{2}+\varepsilon \Delta\right)} Q_{N} \delta * u_{0},
$$

where $\delta$ denotes the Dirac measure. Then, as a consequence of (2.7), of the boundedness of the Riesz transform, and of (3.6), we can write that

$$
\begin{aligned}
\left\|Q_{N} e^{i t\left(\Delta^{2}+\varepsilon \Delta\right)} u_{0}\right\|_{L^{\infty}} & \leq\left\|e^{i t\left(\Delta^{2}+\varepsilon \Delta\right)} Q_{N} \delta\right\|_{L^{\infty}}\left\|u_{0}\right\|_{L^{1}} \\
& \leq N^{-n}\left\||\nabla|^{n} e^{i t\left(\Delta^{2}+\varepsilon \Delta\right)} Q_{N} \delta\right\|_{L^{\infty}}\left\|u_{0}\right\|_{L^{1}} \\
& \leq C N^{-n} \sup _{|\alpha|=n}\left\|D^{\alpha} e^{i t\left(\Delta^{2}+\varepsilon \Delta\right)} Q_{N} \delta\right\|_{L^{\infty}}\left\|u_{0}\right\|_{L^{1}} \\
& \leq C\left|N^{2} t\right|^{-\frac{n}{2}}\left\|u_{0}\right\|_{L^{1}},
\end{aligned}
$$

where $C$ does not depend on $N, u_{0}$ or $t$, and, in case $\varepsilon=1$, we assume $|t| \leq 1$. Now, we can use (3.13) and (3.15) to compute

$$
\begin{aligned}
\left\|\mathcal{V}_{N}(s) \mathcal{V}_{N}(t)^{*} g\right\|_{L^{\infty}} & =\left\|d_{N} Q_{N}^{2} \mathcal{U}(s) \mathcal{U}(t)^{*} d_{N}^{*} g\right\|_{L^{\infty}} \\
& \leq N^{\frac{n}{2}}\left\|Q_{N} e^{i(s-t)\left(\Delta^{2}+\varepsilon \Delta\right)} d_{N}^{*} g\right\|_{L^{\infty}} \\
& \leq C N^{-\frac{n}{2}}|t|^{-\frac{n}{2}}\left\|d_{N}^{*} g\right\|_{L^{1}} \\
& \leq C|t|^{-\frac{n}{2}}\|g\|_{L^{1}}
\end{aligned}
$$

By (3.14) and (3.16), we can apply the results of Keel and Tao [16] to the operators $\mathcal{V}_{N}(t)$. We then get that for any S-admissible pairs $(q, r)$ and $(a, b)$, the following holds true:

$$
\begin{aligned}
& \left\|d_{N} Q_{N} \mathcal{U}(t) u_{0}\right\|_{L^{q}\left(\mathbb{R}, L^{r}\right)} \leq C\left\|u_{0}\right\|_{L^{2}}, \text { and } \\
& \left\|d_{N} \int_{s<t} Q_{N}^{2} \mathcal{U}(t-s) h(s) d s\right\|_{L^{q}\left(\mathbb{R}, L^{r}\right)} \leq C\left\|d_{N} h\right\|_{L^{a^{\prime}}\left(\mathbb{R}, L^{b^{\prime}}\right)} .
\end{aligned}
$$


Now, applying the first inequality of (3.17) to $P_{N} u_{0}$, the second inequality of (3.17) to $P_{N} h$, and considering the effect of dilations $d_{N}$ on space norms, we get

$$
\begin{aligned}
& N^{\frac{n}{2}-\frac{n}{r}}\left\|P_{N} \mathcal{U}(t) u_{0}\right\|_{L^{q}\left(\mathbb{R}, L^{r}\right)} \leq C\left\|P_{N} u_{0}\right\|_{L^{2}}, \text { and } \\
& N^{\frac{n}{2}-\frac{n}{r}}\left\|\int_{s<t} P_{N} \mathcal{U}(t-s) h(s) d s\right\|_{L^{q}\left(\mathbb{R}, L^{r}\right)} \leq C N^{\frac{n}{2}-\frac{n}{b^{\prime}}}\left\|P_{N} h\right\|_{L^{a^{\prime}}\left(\mathbb{R}, L^{b^{\prime}}\right)} .
\end{aligned}
$$

At this point, using (2.7), (3.8), (3.12), (3.18), and the Littlewood-Paley Theorem, we prove (3.11) by writing that

$$
\begin{aligned}
\|u\|_{L^{q}\left(I, L^{r}\right)} & \leq C\left\|\left(\sum_{N}\left|P_{N} u\right|^{2}\right)^{\frac{1}{2}}\right\|_{L^{q}\left(I, L^{r}\right)} \\
& \leq C\left(\sum_{N}\left\|P_{N}\left(e^{i t\left(\Delta^{2}+\varepsilon \Delta\right)} u_{0}+i \int_{0}^{t} e^{i(t-s)\left(\Delta^{2}+\varepsilon \Delta\right)} h(s) d s\right)\right\|_{L^{q}\left(I, L^{r}\right)}^{2}\right)^{\frac{1}{2}} \\
& \leq C\left(\sum_{N} N^{-\frac{4}{q}}\left\|P_{N} u_{0}\right\|_{L^{2}}^{2}+N^{-\frac{4}{q}-\frac{4}{a}}\left\|P_{N} \mathbf{1}_{I} h\right\|_{L^{a^{\prime}}\left(\mathbb{R}, L^{b^{\prime}}\right)}^{2}\right)^{\frac{1}{2}} \\
& \leq C\left(\sum_{N}\left\||\nabla|^{-\frac{2}{q}} P_{N} u_{0}\right\|_{L^{2}}^{2}\right)^{\frac{1}{2}}+C\left(\sum_{N}\left\||\nabla|^{-\frac{2}{q}-\frac{2}{a}} P_{N} \mathbf{1}_{I} h\right\|_{L^{a^{\prime}}\left(\mathbb{R}, L^{b^{\prime}}\right)}^{2}\right)^{\frac{1}{2}} \\
& \leq C\left\|u_{0}\right\|_{\dot{H}^{-\frac{2}{q}}}+\left\||\nabla|^{-\frac{2}{q}-\frac{2}{a}} h\right\|_{L^{a^{\prime}}\left(I, L^{b^{\prime}}\right)}
\end{aligned}
$$

where $\mathbf{1}_{I}$ stands for the characteristic function of $I$, and summation in the above inequalities occurs over all dyadic integers $N$. This ends the proof of (3.11) and of Proposition 3.1.

A direct consequence of (3.11) and Sobolev's inequality is that when $n \geq 5$, for any $B$-admissible pair $(q, r)$, if $u \in C\left(I, H^{-4}\right)$ solves (3.1) with $u_{0} \in \dot{H}^{2}$ and $h \in N(I)$, then $u \in C\left(I, \dot{H}^{2}\right) \cap M(I)$, where $N(I)$ and $M(I)$ are defined in (2.3), and

$$
\|\Delta u\|_{L^{q}\left(I, L^{r}\right)} \leq C\left(\left\|\Delta u_{0}\right\|_{L^{2}}+\|\nabla h\|_{L^{2}\left(I, L^{\frac{2 n}{n+2}}\right)}\right) .
$$

A key feature of (3.19) is that the second derivative of $u$ in the left hand side of (3.19) is estimated using only one derivative of the forcing term $h$. Note that for classical second order Schrödinger equations, estimates like (3.11) and (3.19) do not hold true as they would violate Galilean invariance.

Another estimate we need when discussing scattering in Section 9 is stated as follows.

Proposition 3.2. Let $\varepsilon=-1$ or $\varepsilon=0$. Let also $(a, b)$ and $(q, r)$ be $S$ admissible pairs, $s \geq 0$, and $h \in L^{a^{\prime}}\left(\mathbb{R}, \dot{H}^{s-\frac{2}{a}, b^{\prime}}\right)$. Then

$$
\left\|\int_{\mathbb{R}} e^{-i t\left(\Delta^{2}+\varepsilon \Delta\right)} h(t) d t\right\|_{\dot{H}^{s}} \leq C\left\||\nabla|^{s-\frac{2}{a}} h\right\|_{L^{a^{\prime}}\left(\mathbb{R}, L^{b^{\prime}}\right)},
$$

where $C>0$ depends only on the dimension, and $a^{\prime}, b^{\prime}$ are the conjugate exponents of $a$ and $b$. 
Proof. Again, we may assume $s=0$. For $N$ a dyadic number, we let $\mathcal{V}_{N}(t)$ be as in (3.13). Applying the result in Keel and Tao [16], thanks to (3.14) and (3.16), we get that

$$
\begin{aligned}
\left\|\int_{\mathbb{R}} Q_{N} e^{-i s\left(\Delta^{2}+\varepsilon \Delta\right)} h(s) d s\right\|_{L^{2}} & =\left\|\int_{\mathbb{R}} \mathcal{V}_{N}(s)^{*} d_{N} h(s) d s\right\|_{L^{2}} \\
& \leq C\left\|d_{N} h\right\|_{L^{a^{\prime}}\left(\mathbb{R}, L^{b^{\prime}}\right)} \\
& =N^{\frac{n}{2}-\frac{n}{b^{\prime}}\|h\|_{L^{a^{\prime}}\left(\mathbb{R}, L^{b^{\prime}}\right)}}
\end{aligned}
$$

Applying estimates (3.21) to $P_{N} h$, using (2.7), and the Littlewood-Paley theorem, we finally get

$$
\begin{aligned}
\left\|\int_{\mathbb{R}} e^{-i s\left(\Delta^{2}+\varepsilon \Delta\right)} h(s) d s\right\|_{L^{2}}^{2} & \leq C \sum_{N}\left\|P_{N} \int_{\mathbb{R}} e^{-i s\left(\Delta^{2}+\varepsilon \Delta\right)} h(s) d s\right\|_{L^{2}}^{2} \\
& \leq C \sum_{N} N^{-\frac{2}{a}}\left\|P_{N} h\right\|_{L^{a^{\prime}}\left(\mathbb{R}, L^{b^{\prime}}\right)}^{2} \\
& \leq C\left\||\nabla|^{-\frac{2}{a}} h\right\|_{L^{a^{\prime}}\left(\mathbb{R}, L^{b^{\prime}}\right)}^{2},
\end{aligned}
$$

where the sum in (3.22) is over all dyadic integers $N$. This ends the proof of Proposition 3.2.

\section{Local and Global Existence in the subcritical case}

For the reader's convenience we very briefly discuss the local and global theory for (1.2) in the subcritical regime where $1<p<2^{\sharp}$ if $n \geq 5$, and $1<p<\infty$ if $n \leq 4$. Most of the results in this section go back to Fibich, Ilan, and Papanicolaou [5]. They can be seen as a direct consequence of the straightforward Strichartz estimates (3.10) of Proposition 3.1. As a preliminary remark, it can be noted that for $\alpha>0$, the scaling

$$
u(t, x) \rightarrow \alpha^{\frac{4}{p-1}} u\left(\alpha^{4} t, \alpha x\right)
$$

preserves (1.2) when $\varepsilon=0$, and that letting $\alpha=|\varepsilon|^{-1 / 2}$, (4.1) transforms a solution of (1.2) with $\varepsilon \neq 0$ into a solution of (1.2) with $|\varepsilon|=1$. In particular, we may assume in what follows that $\varepsilon \in\{-1,0,1\}$. As another easy remark, it can be noted that equations like (1.1) also enjoy time reversal symmetry, and time translation symmetry. Unless otherwise stated, $\lambda$ and $u_{0}$ in this section are arbitrary.

Proposition 4.1. Given any initial data $u_{0} \in H^{2}$, any $p \in\left(1,2^{\sharp}-1\right)$ when $n \geq 5$, and any $p>1$ when $n \leq 4$, there exists $T>0$ and a unique solution $u \in C\left([0, T], H^{2}\right)$ of $(1.2)$ such that $u(0)=u_{0}$. The solution has conserved mass and energy in the sense that

$$
M(u(t))=M\left(u_{0}\right) \text { and } E(u(t))=E\left(u_{0}\right)
$$

for all $t \in[0, T]$, where the mass $M$ is defined in (2.1), and the energy $E$ is defined in (2.2). Besides, if $T^{*}$ is the maximal time of existence of $u$, then

$$
\lim _{t \rightarrow T^{*}}\|u(t)\|_{H^{2}}=+\infty
$$

when $T^{*}<+\infty$, and the solution map $u_{0} \rightarrow u$ is continuous in the sense that for any $T \in\left(0, T^{*}\right)$, if $u_{0}^{k} \in H^{2}$ is a sequence converging in $H^{2}$ to $u_{0}$, and if $u^{k}$ denotes the solution of (1.2) with initial data $u_{0}^{k}$, then $u^{k}$ is defined on $[0, T]$ for sufficiently large $k$ and $u^{k} \rightarrow u$ in $C\left([0, T], H^{2}\right)$. 
Proof. Proposition 4.1 follows from an easy adaptation of the standard proof for second order Schrödinger equations, as developed, for instance, in Cazenave [3], once the Strichartz estimates (3.10) have been established.

A direct consequence of Proposition 4.1 is as follows.

Corollary 4.1. Let $p \in\left(1,2^{\sharp}-1\right)$ when $n \geq 5, p>1$ when $n \leq 4, u_{0} \in H^{2}$, and $u$ be the solution of (1.2) with initial data $u_{0}$. Then $u$ can be extended to a solution on the whole of $\mathbb{R}$ in the following cases:

(a) $\lambda \geq 0$,

(b) $\lambda<0$ and $p<1+\frac{8}{n}$,

(c) $\lambda<0, p=1+\frac{8}{n}$ and $u_{0}$ is sufficiently small in $L^{2}$,

(d) $\lambda<0$ and $u_{0}$ is sufficiently small in $H^{2}$.

In particular, when $n \geq 5$, for any $u_{0} \in H^{2}$ there exists a unique global solution $u \in C\left(\mathbb{R}, H^{2}\right)$ of (1.2) such that $u(0)=u_{0}$ if $\lambda>0$ and $p \in\left(1,2^{\sharp}-1\right)$.

Proof. The first assertion directly follows from conservation of energy. The second and third assertions follow from Gagliardo-Nirenberg's inequalities and conservation of mass and energy. Indeed,

$$
\begin{aligned}
\int_{\mathbb{R}^{n}}|\Delta u(t, x)|^{2} d x & \leq E\left(u_{0}\right)+\varepsilon \int_{\mathbb{R}^{n}}|\nabla u(t, x)|^{2} d x-\frac{2 \lambda}{p+1} \int_{\mathbb{R}^{n}}|u(t, x)|^{p+1} d x \\
& \leq E\left(u_{0}\right)+\left\|u_{0}\right\|_{L^{2}}\|\Delta u\|_{L^{2}}+C\left\|u_{0}\right\|_{L^{2}}^{p+1-\frac{n(p-1)}{4}}\|\Delta u\|_{L^{2}}^{\frac{n(p-1)}{4}} .
\end{aligned}
$$

and, when $p<1+\frac{8}{n}$, or when $p=1+\frac{8}{n}$ and $\left\|u_{0}\right\|_{L^{2}}$ is sufficiently small, we get a contradiction with (4.3) if $T^{*}<+\infty$. The last assertion follows from a Payne and Sattinger [21] type argument similar to the one developed in the proof of Corollary 5.1 in Section 5.

Following the strategy in Lin and Strauss [19], see also Cazenave [3], we can prove that scattering holds true in the whole energy space $H^{2}$ when $\lambda \geq 0, n \geq 5$, $\varepsilon \leq 0$, and $1+\frac{8}{n}<p<2^{\sharp}-1$. We also refer to Guo and Wang [9] and Wang [30] for similar considerations in the small norm setting.

\section{Local existence in the critical case}

We briefly develop the local theory for (1.2) in the energy critical case. Here $p=2^{\sharp}-1$ and $n \geq 5$. As in the preceding section, $\lambda$ and $u_{0}$ can be arbitrary. If $u \in C\left(I, H^{2}\right)$ is a solution of the critical equation (1.2) with initial data $u_{0}$, then

$$
u(t)=e^{i t\left(\Delta^{2}+\varepsilon \Delta\right)} u_{0}+i \lambda \int_{0}^{t} e^{i(t-s)\left(\Delta^{2}+\varepsilon \Delta\right)}|u(s)|^{\frac{8}{n-4}} u(s) d s .
$$

Conversely, if $u_{0} \in H^{2}$, and $u \in W(I)$ solves (5.1), then $|u|^{\frac{8}{n-4}} u \in N(I)$, where $W(I)$ and $N(I)$ are defined in Section $2, u \in C\left(I, H^{2}\right)$ by the Strichartz estimates (3.10) and (3.19), and $u$ is a solution of the critical (1.2) with initial data $u_{0}$. Equations like (5.1) are often referred to as Duhamel's formula. Here again, because of the scaling invariance (4.1) we may assume that $\varepsilon \in\{-1,0,1\}$. Local existence is settled by Proposition 5.1. Stability, and uniform continuity of the map $u_{0} \mapsto u$, are discussed in the following section. 
Proposition 5.1. Let $n \geq 5$ and $p=2^{\sharp}-1$. There exists $\delta>0$ such that for any initial data $u_{0} \in H^{2}$, and any interval $I=[0, T]$ with $T \leq 1$ when $\varepsilon=1$, if

$$
\left\|e^{i t\left(\Delta^{2}+\varepsilon \Delta\right)} u_{0}\right\|_{W(I)}<\delta,
$$

where $W(I)$ is as in (2.3) in Section 2, then there exists a unique solution $u \in$ $C\left(I, H^{2}\right)$ of (1.2) with initial data $u_{0}$. This solution has conserved mass and energy in the sense of (4.2), and satisfies $u \in M(I) \cap L^{\frac{2(n+4)}{n}}\left(I \times \mathbb{R}^{n}\right)$. Moreover,

$$
\begin{aligned}
& \|u\|_{W(I)} \leq 2 \delta, \text { and } \\
& \|u\|_{M(I)}+\|u\|_{L^{\infty}\left(I, H^{2}\right)} \leq C\left(\left\|u_{0}\right\|_{H^{2}}+\delta^{\frac{n+4}{n-4}}\right)
\end{aligned}
$$

for some $C>0$ independent of $u_{0}$. Besides, the solution depends continuously on the initial data in the sense that there exists $\delta_{0}$, depending on $\delta$, such that, for any $\delta_{1} \in\left(0, \delta_{0}\right)$, if $\left\|v_{0}-u_{0}\right\|_{H^{2}} \leq \delta_{1}$, and if we let $v$ be the local solution of (1.2) with initial data $v_{0}$, then $v$ is defined on $I$ and

$$
\|u-v\|_{L^{q}\left(I, L^{r}\right)} \leq C \delta_{1},
$$

for any $B$-admissible pair $(q, r)$ in the sense of $(3.9)$, where $C>0$ is independent of $u_{0}$ and $v_{0}$.

Proof. The proposition follows from a contraction mapping argument. For $u \in W(I)$, we let $\Phi_{u_{0}}(u)$ be given by the right hand side in (5.1). Thanks to the Strichartz estimates (3.10) and (3.19) stated after the proof of Proposition 3.1, $\Phi_{u_{0}}$ is a contraction on the set

$$
X_{M, \delta}=\left\{v \in M(I) ;\|v\|_{W(I)} \leq 2 \delta,\|u\|_{L} \frac{2(n+4)}{n}\left(I, L \frac{2(n+4)}{n}\right) \leq 2 M\right\}
$$

for $M=C\left\|u_{0}\right\|_{L^{2}}$, and $\delta>0$ sufficiently small, where we equip $X_{M, \delta}$ with the $L^{\frac{2(n+4)}{n}}\left(I, L^{\frac{2(n+4)}{n}}\right)$ norm. The contraction mapping theorem gives a unique solution $u$ in $X_{M, \delta}$, and a standard variant of the argument gives (5.4). The Strichartz estimates (3.19) give that $u \in M(I) \cap L^{\infty}\left(I, H^{2}\right)$ and (5.3). A straightforward adaptation of Cazenave [3, Chapter 4] gives uniqueness in $C\left([0, T], \dot{H}^{2}\right)$ and conservation of mass and energy.

As a remark, for any $u_{0} \in H^{2},(5.2)$ holds true for $T>0$ sufficiently small. Global existence for small data in the energy space, as mentioned in the remark after Theorem 1.1, is a direct consequence of Proposition 5.1.

Corollary 5.1. Let $n \geq 5$ and $p=2^{\sharp}-1$. There exists $\epsilon_{0}>0$ such that for any $u_{0} \in H^{2}$ satisfying $\mathcal{E}\left(u_{0}\right) \leq \epsilon_{0}$, where $\mathcal{E}$ is as in (2.4), equation (1.2) possesses a unique solution $u \in C\left(\mathbb{R}, H^{2}\right)$ with initial data $u_{0}$.

Proof. By the Strichartz estimates (3.19), we see that if $u$ exists on $\left[0, t_{0}\right]$, and if the $\dot{H}^{2}$-norm of $u\left(t_{0}\right)$ is sufficiently small, then we can use (5.2) to extend $u$ on $\left[t_{0}, t_{0}+1\right]$. Hence, in order to prove global existence, it suffices to prove that $\|u(t)\|_{\dot{H}^{2}}$ remains small on the whole interval of existence of $u$. We prove this now. Let $t>0$ be such that $u$ is defined on $[0, t]$. By conservation of energy and Sobolev's inequality we can write that

$$
E(u(t))=E\left(u_{0}\right) \leq C\left(\mathcal{E}\left(u_{0}\right)+\mathcal{E}\left(u_{0}\right)^{2^{\sharp} / 2}\right) .
$$


When $\lambda>0$, global existence follows from (5.5) since $\|u(t)\|_{\dot{H}^{2}}^{2} \leq E(u(t))$. When $\lambda<0$, we write with conservation of energy and Sobolev's inequality that

$$
\begin{aligned}
\|u(t)\|_{\dot{H}^{2}}^{2} & \leq 2 E\left(u_{0}\right)+|\varepsilon|\|u(t)\|_{L^{2}}\|u\|_{\dot{H}^{2}}+|\lambda| \frac{n-4}{n}\|u(t)\|_{L^{2^{\sharp}}}^{2^{\sharp}} \\
& \leq C\left(\mathcal{E}\left(u_{0}\right)+\mathcal{E}\left(u_{0}\right)^{2^{\sharp} / 2}\right)+\mathcal{E}\left(u_{0}\right)^{\frac{1}{2}}\|u\|_{\dot{H}^{2}}+C\|u(t)\|_{\dot{H}^{2}}^{2^{\sharp}} \\
& \leq C\left(\mathcal{E}\left(u_{0}\right)+\mathcal{E}\left(u_{0}\right)^{2^{\sharp} / 2}\right)+\mathcal{E}\left(u_{0}\right)+\frac{1}{4}\|u\|_{\dot{H}^{2}}^{2}+C\|u(t)\|_{\dot{H}^{2}}^{2^{\sharp}} .
\end{aligned}
$$

Here again it follows from $(5.6)$ that if $\mathcal{E}\left(u_{0}\right)$ is sufficiently small, then $u$ stays small in the $\dot{H}^{2}$-norm.

In order to end the section we now discuss a useful criterion for global existence.

Proposition 5.2. Let $n \geq 5$ and $p=2^{\sharp}-1$. Let $u \in C\left([0, T), H^{2}\right)$ be a solution of (1.2) such that $\|u\|_{Z([0, T])}<+\infty$. Then there exists $K=K\left(\left\|u_{0}\right\|_{H^{2}},\|u\|_{Z([0, T])}\right)$, respectively $K=K\left(T,\left\|u_{0}\right\|_{H^{2}},\|u\|_{Z([0, T])}\right)$ when $\varepsilon>0$, such that

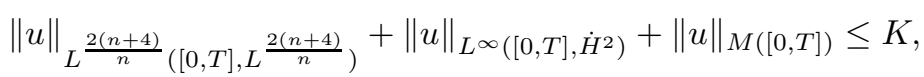

and $u$ can be extended to a solution $\tilde{u} \in C\left(\left[0, T^{\prime}\right), H^{2}\right)$ of (1.2) for some $T^{\prime}>T$.

Proof. Let $\eta>0$ be small. Let also $B=\|u\|_{Z([0, T])}$. For $x \geq 0$ we let $[x]$ be the largest integer not exceeding $x$. If $\varepsilon \leq 0$, we split [0,T] into

$$
N=\left[(B / \eta)^{\frac{2(n+4)}{n-4}}\right]+1
$$

intervals $I_{j}, j=1 \ldots N$, such that for $1 \leq j \leq N-1,\|u\|_{Z\left(I_{j}\right)}=\eta$, and $\|u\|_{Z\left(I_{N}\right)} \leq$ $\eta$. If $\varepsilon=1$, we split $[0, T]$ into $N$ intervals $I_{j}, j=1 \ldots N$, for which $\|u\|_{Z\left(I_{j}\right)} \leq \eta$ and $\left|I_{j}\right| \leq 1$, one of these inequalities being an equality if $j<N$. Then,

$$
N \leq|T|+3+\left[(B / \eta)^{\frac{2(n+4)}{n-4}}\right] .
$$

Applying the Strichartz estimates (3.19) in $I_{j}=\left[t_{j}, t_{j+1}\right]$, we get, for $t \in I_{j}$,

$$
\begin{aligned}
\|u\|_{M\left(\left[t_{j}, t\right]\right)} & \leq C\left\|u\left(t_{j}\right)\right\|_{\dot{H}^{2}}+C\left\||u|^{\frac{8}{n-4}} u\right\|_{N\left(\left[t_{j}, t\right]\right)} \\
& \leq C\left\|u\left(t_{j}\right)\right\|_{\dot{H}^{2}}+C\|u\|_{Z\left(I_{j}\right)}^{\frac{8}{n-4}}\|u\|_{M\left(\left[t_{j}, t\right]\right)} \\
& \leq C\left\|u\left(t_{j}\right)\right\|_{\dot{H}^{2}}+C \eta^{\frac{8}{n-4}}\|u\|_{M\left(\left[t_{j}, t\right]\right)},
\end{aligned}
$$

where $C>0$ depends only on $n$ and $\lambda$. Applying the Strichartz estimates (3.10) in $I_{j}$, and conservation of mass, we get that

$$
\begin{aligned}
\|u\|_{L} \frac{2(n+4)}{n}\left(\left[t_{j}, t\right], L \frac{2(n+4)}{n}\right) & \leq C\left\|u\left(t_{j}\right)\right\|_{L^{2}}+C\left\|\left.u\right|^{\frac{8}{n-4}} u\right\|_{L^{\frac{2(n+4)}{n+8}}\left(\left[t_{j}, t\right] \times \mathbb{R}^{n}\right)} \\
& \leq C\left\|u_{0}\right\|_{L^{2}}+C\|u\|_{Z\left(\left[t_{j}, t\right]\right)}^{\frac{8}{n-4}}\|u\|_{L} \frac{2(n+4)}{n}\left(\left[t_{j}, t\right], L \frac{2(n+4)}{n}\right) \\
& \leq C\left\|u_{0}\right\|_{L^{2}}+C \eta^{\frac{8}{n-4}}\|u\|_{L}^{\frac{2(n+4)}{n}\left(\left[t_{j}, t\right], L \frac{2(n+4)}{n}\right)} .
\end{aligned}
$$

If $\eta$ is sufficiently small, (5.9) implies that

$$
\|u\|_{L} \frac{2(n+4)}{n}\left(I_{j}, L \frac{2(n+4)}{n}\right) \leq C\left\|u_{0}\right\|_{L^{2}}
$$


while (5.8) implies that $\|u\|_{M\left(I_{j}\right)} \leq 2 C\left\|u\left(t_{j}\right)\right\|_{\dot{H}^{2}}$. Applying again the Strichartz estimates (3.19) this gives $\|u\|_{L^{\infty}\left(I_{j}, \dot{H}^{2}\right)} \leq 2 C\left\|u\left(t_{j}\right)\right\|_{\dot{H}^{2}}$. In particular, $\left\|u\left(t_{j+1}\right)\right\|_{\dot{H}^{2}} \leq$ $2 C\left\|u\left(t_{j}\right)\right\|_{\dot{H}^{2}}$, and finally,

$$
\begin{aligned}
& \left.\|u\|_{L^{\frac{2(n+4)}{n}}([0, T), L} \frac{2(n+4)}{n}\right) \\
& \|u\|_{L^{\infty}\left([0, T), \dot{H}^{2}\right)} \leq(2 C)^{N}\left\|u_{0}\right\|_{\dot{H}^{2}}<+\infty, \text { and } \\
& \|u\|_{M\left(I_{j}\right)} \leq(2 C)^{N}\left\|u_{0}\right\|_{\dot{H}^{2}}
\end{aligned}
$$

for all $j$. By (5.10) we get that (5.7) holds true. Now, let $t_{0} \in I_{N}$. Duhamel's formula (5.1) gives that

$$
u(t)=e^{i\left(t-t_{0}\right)\left(\Delta^{2}+\varepsilon \Delta\right)} u\left(t_{0}\right)+i \lambda \int_{t_{0}}^{t} e^{i(t-s)\left(\Delta^{2}+\varepsilon \Delta\right)}|u(s)|^{\frac{8}{n-4}} u(s) d s
$$

for all $t$, and (5.11), Sobolev's inequality, and the Strichartz estimates (3.19) give

$$
\begin{aligned}
\left\|e^{i\left(t-t_{0}\right)\left(\Delta^{2}+\varepsilon \Delta\right)} u\left(t_{0}\right)\right\|_{W\left(\left[t_{0}, T\right]\right)} & \leq\|u\|_{W\left(\left[t_{0}, T\right]\right)}+\left.C\|\| u\right|^{\frac{8}{n-4}} u \|_{N\left(\left[t_{0}, T\right]\right)} \\
& \leq\|u\|_{W\left(\left[t_{0}, T\right]\right)}+C\|u\|_{W\left(\left[t_{0}, T\right]\right)}^{\frac{n+4}{n-4}} .
\end{aligned}
$$

Since the $W([0, T])$-norm of $u$ is finite, dominated convergence ensures that the $W\left(\left[t_{0}, T\right]\right)$-norm of $u$ can be made arbitrarily small as $t_{0} \rightarrow T$, and (5.12) shows that the $W\left(\left[t_{0}, T\right]\right)$-norm of the free propagator

$$
t \mapsto e^{i\left(t-t_{0}\right)\left(\Delta^{2}+\varepsilon \Delta\right)} u\left(t_{0}\right)
$$

is like $o(1)$ as $t_{0}$ tends to $T$. In particular, we can find $t_{1} \in(0, T)$ and $T^{\prime}>T$ such that $u\left(t_{1}\right) \in H^{2}$ and

$$
\left\|e^{i\left(t-t_{1}\right)\left(\Delta^{2}+\varepsilon \Delta\right)} u\left(t_{1}\right)\right\|_{W\left(\left[t_{1}, T^{\prime}\right]\right)} \leq \delta .
$$

Now, it follows from (5.13) and Proposition 5.1 that there exists $v \in C\left(\left[t_{1}, T^{\prime}\right], H^{2}\right)$ such that $v$ solves (1.2) with $p=2^{\sharp}-1$ and $v\left(t_{1}\right)=u\left(t_{1}\right)$. By uniqueness, $u=v$ in $\left[t_{1}, T\right)$ and $u$ can be extended in $\left[0, T^{\prime}\right]$. This ends the proof of Proposition 5.2.

As a direct consequence of Proposition 5.2, if $T^{*}$ is the maximal time of existence of $u$, and $T^{*}<+\infty$, then, necessarily, $\|u\|_{Z\left(\left[0, T^{*}\right]\right)}=+\infty$.

\section{Stability in the critical case}

We briefly discuss stability in the energy critical case of (1.2) following the approach developed by Tao and Visan [28] in the case of the energy critical second order Schrödinger equation. Stability is of importance for physical considerations if one keeps in mind that equations like (1.2) are often mathematical approximations of more physically relevant equations, as pointed out in Fibich, Ilan, and Papanicolaou [5]. Stability, in its global version, can be stated as follows. As in the preceding sections, $\lambda$ and $u_{0}$ can be made arbitrary, and we may assume that $\varepsilon \in\{-1,0,1\}$.

Proposition 6.1. Let $n \geq 5$ and $p=2^{\sharp}-1$. Let $I \subset \mathbb{R}$ be a compact time interval such that $0 \in I$, and $\tilde{u}$ be an approximate solution of (1.2) in the sense that

$$
i \partial_{t} \tilde{u}+\Delta^{2} \tilde{u}+\varepsilon \Delta \tilde{u}+\lambda|\tilde{u}|^{\frac{8}{n-4}} \tilde{u}=e
$$

for some $e \in N(I)$. Assume that $\|\tilde{u}\|_{Z(I)}<+\infty$ and $\|\tilde{u}\|_{L^{\infty}\left(I, \dot{H}^{2}\right)}<+\infty$. For any $\Lambda>0$ there exists $\delta_{0}>0, \delta_{0}=\delta_{0}\left(\Lambda,\|\tilde{u}\|_{Z(I)},\|\tilde{u}\|_{L^{\infty}\left(I, \dot{H}^{2}\right)}\right)$ if $\varepsilon \leq 0$, and 
$\delta_{0}=\delta_{0}\left(\Lambda,\|\tilde{u}\|_{Z(I)},\|\tilde{u}\|_{L^{\infty}\left(I, \dot{H}^{2}\right)},|I|\right)$ if $\varepsilon>0$, such that if $\|e\|_{N(I)} \leq \delta$, and $u_{0} \in H^{2}$ satisfies

$$
\left\|\tilde{u}(0)-u_{0}\right\|_{\dot{H}^{2}} \leq \Lambda \text { and }\left\|e^{i t\left(\Delta^{2}+\varepsilon \Delta\right)}\left(\tilde{u}(0)-u_{0}\right)\right\|_{W(I)} \leq \delta
$$

for some $\delta \in\left(0, \delta_{0}\right]$, then there exists $u \in C\left(I, H^{2}\right)$ a solution of (1.2) such that $u(0)=u_{0}$. Moreover, $u$ satisfies

$$
\begin{aligned}
& \|u-\tilde{u}\|_{W(I)} \leq C\left(\delta+\delta^{\frac{15}{(n-4)^{2}}}\right), \\
& \|u-\tilde{u}\|_{L^{q}\left(I, \dot{H}^{2, r}\right)} \leq C\left(\Lambda+\delta+\delta^{\frac{15}{(n-4)^{2}}}\right), \text { and } \\
& \|u\|_{L^{q}\left(I, \dot{H}^{2, r}\right)} \leq C
\end{aligned}
$$

for all B-admissible pairs $(q, r)$, where $C=C\left(\Lambda,\|\tilde{u}\|_{Z(I)},\|\tilde{u}\|_{L^{\infty}\left(I, \dot{H}^{2}\right)}\right)$ if $\varepsilon \leq 0$, and $C=C\left(\Lambda,\|\tilde{u}\|_{Z(I)},\|\tilde{u}\|_{L^{\infty}\left(I, \dot{H}^{2}\right)},|I|\right)$ if $\varepsilon>0$, are nondecreasing functions of their arguments.

Letting $e=0$ in Proposition 6.1 provides the uniform continuity of the solution map $u_{0} \rightarrow u$. On such a statement, recall that by the Strichartz estimates (3.19),

$$
\left\|e^{i t\left(\Delta^{2}+\varepsilon \Delta\right)}\left(\tilde{u}(0)-u_{0}\right)\right\|_{W(I)} \leq C\left\|\tilde{u}(0)-u_{0}\right\|_{\dot{H}^{2}} .
$$

In particular we can take $\delta=C \Lambda$ in (6.2) and make $\delta$ small when $\Lambda$ is small. Proposition 6.1 is an easy consequence of the Strichartz estimates (3.19) when $n \leq 12$, and is more delicate to prove when $n>12$. We briefly sketch the proof when $n \leq 12$, and refer to Tao and Visan [28] with only very little indications on the proof when $n>12$. As a first claim, because Proposition 6.1 can be localized, we may assume, see Tao and Visan [28] for the argument in the second order case, that $\|\tilde{u}\|_{W(I)} \leq \delta$ and that $|I| \leq 1$ when $\varepsilon>0$. Let $f$ be given by $f(z)=\lambda|z|^{2^{\sharp}}-2 z$ for $z \in \mathbb{C}$. Assuming that $n \leq 12$, letting $v=u-\tilde{u}$, where $u$ solves (1.2) with initial data $u_{0}$, and $I^{\prime}$ be the maximal time interval of existence of $u$, we can write, using (6.1), that

$$
i \partial_{t} v+\Delta^{2} v+\varepsilon \Delta v+f(\tilde{u}+v)-f(\tilde{u})=e
$$

in $I \cap I^{\prime}$. The Strichartz estimates (3.19) then give that for $t \geq 0$ such that $I_{t}=[0, t] \subset I$,

$$
\begin{aligned}
\|v\|_{W\left(I_{t}\right)} & \leq C\left\|e^{i\left(t-t_{0}\right)\left(\Delta^{2}+\varepsilon \Delta\right)}\left(\tilde{u}\left(t_{0}\right)-u_{0}\right)\right\|_{W\left(I_{t}\right)} \\
& +C\|f(\tilde{u}+v)-f(\tilde{u})\|_{N\left(I_{t}\right)}+C\|e\|_{N\left(I_{t}\right)} \\
& \leq 2 C \delta+C\left\|\left(f_{z}(\tilde{u}+v)-f_{z}(\tilde{u})\right) \nabla \tilde{u}\right\|_{L^{2}\left(I_{t}, L^{\frac{2 n}{n+2}}\right)} \\
& +C\left\|\left(f_{\bar{z}}(\tilde{u}+v)-f_{\bar{z}}(\tilde{u})\right) \nabla \overline{\tilde{u}}\right\|_{L^{2}\left(I_{t}, L^{\frac{2 n}{n+2}}\right)} \\
& +C\left\|f_{z}(\tilde{u}+v) \nabla v\right\|_{L^{2}\left(I_{t}, L^{\frac{2 n}{n+2}}\right)} \\
& +C\left\|f_{\bar{z}}(\tilde{u}+v) \nabla \bar{v}\right\|_{L^{2}\left(I_{t}, L^{\frac{2 n}{n+2}}\right)} \\
& \leq C\left(\delta+\delta^{\frac{8}{n-4}}\|v\|_{W\left(I_{t}\right)}+\|v\|_{W\left(I_{t}\right)}^{\frac{n+4}{n-4}}\right)
\end{aligned}
$$

where $f_{z}, f_{\bar{z}}$ are the usual complex derivatives. Noting that $g(t)=\|v\|_{I_{t}}$ defines a continuous function such that $g(0)=0$, and since by (6.5),

$$
g(t) \leq C \delta+C \delta^{\frac{8}{n-4}} g(t)+C g(t)^{\frac{n+4}{n-4}},
$$


we conclude that if $\delta \leq \delta_{0}$ is sufficiently small, depending only on $n$ and $\lambda$, then, for all $t \in I \cap I^{\prime}, g(t) \leq C \delta$ for some positive constant $C$. In particular, the $W\left(I \cap I^{\prime}\right)$-norm of $u$ is bounded and Proposition 5.2 gives that $I \cap I^{\prime}=I$. Another application of the Strichartz estimates (3.19) then gives the control equations (6.3). When $n>12$, the proof of Proposition 6.1 becomes very delicate because $\nabla f(u)$ is no longer lipschitz continuous. The solution to this problem, as developed in Tao and Visan [28], is some sort of Exotic Strichartz estimate in order to work with spaces of functions with greater integrability and lesser regularity (in particular, we require that they involve less than $8 /(n-4)$ derivatives $)$, but which still remain scale-invariant. To close the argument, we then need a good chain-rule for fractional derivatives as proved in Visan [29]. We briefly discuss the proof now. Let $X$ and $Y$ be the norms defined by

$$
\begin{aligned}
& \|u\|_{X(I)}=\left\||\nabla|^{\frac{8 n}{n^{2}-16}} u\right\|_{L^{\frac{n^{2}-16}{8}}\left(I, L \frac{2(n+4)}{n}\right)}, \text { and } \\
& \|u\|_{Y(I)}=\left\||\nabla|^{\frac{8 n}{n^{2}-16}} u\right\|_{L^{\frac{n^{2}-16}{4(n-2)}}\left(I, L^{\frac{2(n+4)}{n+8}}\right)} .
\end{aligned}
$$

These spaces are involved in the following Exotic Strichartz estimates (6.7) which we obtain as a consequence of the result in Foschi [7]. Namely,

$$
\left\|\int_{0}^{t} e^{i(t-s)\left(\Delta^{2}+\varepsilon \Delta\right)} F(s) d s\right\|_{X(I)} \leq C\|F\|_{Y(I)} .
$$

Now, the fact that the $Y$-norm involves less than $8 /(n-4)$ derivative enables us to get the following nonlinear estimate.

Lemma 6.1. Let $n \geq 12$. For any $v \in W(I)$, and any $u \in X(I)$,

$$
\left\|f_{z}(v) u\right\|_{Y(I)} \leq C\|v\|_{W(I)}^{\frac{8}{n-4}}\|u\|_{X(I)}
$$

where $C>0$ depends only on $n$ and $\lambda$. A similar estimate holds true for $f_{\bar{z}}$.

Proof. We use the rule for fractional derivatives of products, see e.g. Kato [15] to estimate

$$
\begin{aligned}
& \left\||\nabla|^{\frac{8 n}{n^{2}-16}}\left(f_{z}(v) u\right)\right\|_{L^{\frac{2(n+4)}{n+8}}} \leq C\left(\left\||\nabla|^{\frac{8 n}{n^{2}-16}} f_{z}(v)\right\|_{L^{\frac{n^{2}-16}{4(n-2)}}}\|u\|_{L^{\frac{2\left(n^{2}-16\right)}{n^{2}-4 n-16}}}\right. \\
& \left.+\left\|f_{z}(v)\right\|_{L \frac{n+4}{4}}\left\||\nabla|^{\frac{8 n}{n^{2}-16}} u\right\|_{L} \frac{2(n+4)}{n}\right) \\
& \leq C\left(\left\||\nabla|^{\frac{8 n}{n^{2}-16}} f_{z}(v)\right\|_{L^{\frac{n^{2}-16}{4(n-2)}}}\left\||\nabla|^{\frac{8 n}{n^{2}-16}} u\right\|_{L} \frac{2(n+4)}{n}\right. \\
& \left.+\|v\|_{L^{\frac{2(n+4)}{n-4}}}^{\frac{8}{n-4}}\left\||\nabla|^{\frac{8 n}{n^{2}-16}} u\right\|_{L} \frac{2(n+4)}{n}\right) .
\end{aligned}
$$

Besides, using the chain-rule for fractional derivatives of fractional powers in Visan [29, Appendix A], the boundedness of Riesz transforms, Hölder's and Sobolev's inequalities, we get

$$
\begin{aligned}
\left\||\nabla|^{\frac{8 n}{n^{2}-16}} f_{z}(v)\right\|_{L^{\frac{n^{2}-16}{4(n-2)}}} & \leq C\left\||v|^{\frac{16}{(n+2)(n-4)}}\right\|_{L}^{\frac{(n+2)(n+4)}{8}}\left\||\nabla| \frac{n+2}{n+4} v\right\|_{L^{\frac{2 n(n+4)}{n^{2}-2 n+4}}}^{\frac{8 n}{(n+2)(n-4)}} \\
& \leq C\|v\|_{L^{\frac{2(n+4)}{n-4}}}^{\frac{16}{(n+2)(n-4)}}\|\nabla v\|_{L^{\frac{2 n(n+4)}{n^{2}-2 n+8}}}^{\frac{8 n}{(n+2)(n-4)}} \\
& \leq C\|\nabla v\|_{L^{\frac{2 n(n+4)}{n^{2}-2 n+8}}}
\end{aligned}
$$


Now, with (6.9), (6.10), and Sobolev's inequality, we get

$$
\left\||\nabla|^{\frac{8 n}{n^{2}-16}}\left(f_{z}(v) u\right)\right\|_{L^{\frac{2(n+4)}{n+8}}} \leq C\|\nabla v\|_{L^{\frac{2 n(n+4)}{n^{2}-2 n+8}}}^{\frac{8}{n-4}}\left\||\nabla|^{\frac{8 n}{n^{2}-16}} u\right\|_{L^{\frac{2(n+4)}{n}}},
$$

and applying Hölder's inequality, we finally get (6.8). This ends the proof of the lemma.

Noting with (6.7) that the $X$-norm of a solution of the linear equation with zero initial data is controlled by the $Y$-norm of its forcing term, we get with (6.8) that the $X$-norm of $\tilde{u}-u$ can be controlled in terms of $\delta$ and $\Lambda$. Once this scale-invariant norm has been controlled, straightforward applications of the Strichartz estimates, as developed in the above mentioned [28], then provide the result.

\section{Almost conservation Laws}

In this section we prove almost conservation of the local mass, and localized Morawetz type estimates. These are important ingredients in the process of proving Theorem 1.1. As in the preceding sections we may assume that $\varepsilon \in\{-1,0,1\}$. First we discuss almost conservation of local mass. Let $\chi \in C_{c}^{\infty}\left(\mathbb{R}^{n}\right)$ be a radially symmetrical smooth nonnegative function such that $\chi(r)=1$ if $r \leq 1, \chi(r)=0$ if $r \geq 2$, and $0 \leq \chi \leq 1$. We define the local mass $M\left(u, B_{x_{0}}(R)\right)$ over the ball $B_{x_{0}}(R)$ of a function $u \in L^{2}$ by

$$
M\left(u, B_{x_{0}}(R)\right)=\int_{\mathbb{R}^{n}}|u(x)|^{2} \chi_{R}^{4}\left(x-x_{0}\right) d x,
$$

where, for ease of exposition, the notation $g_{R}$ when $g$ is a function stands for $g_{R}(x)=g(x / R)$. Note that Hölder's and Sobolev's inequalities give that

$$
M\left(u, B_{x_{0}}(R)\right) \leq C\|\Delta u\|_{L^{2}}^{2} R^{4},
$$

where $C$ depends only on $n$. Now we claim that the local mass of a solution of (1.2) varies slowly in time provided that the radius $R$ is sufficiently large.

Lemma 7.1. Let $p \in\left(1,2^{\sharp}-1\right]$ when $n \geq 5$, and $p>1$ when $n \leq 4$. Let $\lambda \in \mathbb{R}$, possibly zero, and $u \in C\left(I, H^{2}\right)$ be a solution of (1.2). Then

$$
\left|\partial_{t} M\left(u(t), B_{x_{0}}(R)\right)\right| \leq C \frac{\mathcal{E}(u)^{\frac{3}{4}}}{R} M\left(u(t), B_{x_{0}}(R)\right)^{\frac{1}{4}} .
$$

for all $t \in I$, where $C>0$ does not depend on $u$ and $I$.

Proof. By translation symmetry, we can suppose $x_{0}=0$. Integrating by parts, using (1.2), gives

$$
\begin{aligned}
\frac{d}{d t} M\left(u(t), B_{0}(R)\right) & =\frac{16}{R} \operatorname{Re} \int_{\mathbb{R}^{n}} i \Delta u \nabla \bar{u}(\nabla \chi)_{R} \chi_{R}^{3} d x \\
& +\frac{8}{R^{2}} \operatorname{Re} \int_{\mathbb{R}^{n}} i \Delta u\left(\chi_{R}^{3}(\Delta \chi)_{R}+3 \chi_{R}^{2}(\nabla \chi)_{R}^{2}\right) \bar{u} d x \\
& -\frac{8 \varepsilon}{R} \operatorname{Re} \int_{\mathbb{R}^{n}} i \nabla u(\nabla \chi)_{R} \bar{u} \chi_{R}^{3} d x .
\end{aligned}
$$

Now we estimate each term in the right hand side of (7.4) independently one from another. For the first term in the right hand side of (7.4), an application of the 
Cauchy-Schwartz inequality gives

$$
\begin{aligned}
& \left|\int_{\mathbb{R}^{n}} \chi_{R}^{3} \Delta u \nabla \bar{u}(\nabla \chi)_{R} d x\right| \\
& \leq\left\|(\nabla \chi)_{R}\right\|_{L^{\infty}}\left(\int_{\mathbb{R}^{n}}|\Delta u|^{2} d x\right)^{\frac{1}{2}}\left(\int_{\mathbb{R}^{n}}|\nabla u|^{2} \chi_{R}^{6} d x\right)^{\frac{1}{2}} .
\end{aligned}
$$

Integrating by parts,

$$
\int_{\mathbb{R}^{n}}|\nabla u|^{2} \chi_{R}^{6} d x=-\operatorname{Re} \int_{\mathbb{R}^{n}} \bar{u}\left(\Delta u \chi_{R}^{6}+\frac{6}{R} \nabla u(\nabla \chi)_{R} \chi_{R}^{5}\right) d x
$$

and by using Hölder's and Sobolev's inequalities we get that

$$
\begin{aligned}
& \int_{\mathbb{R}^{n}}|\nabla u|^{2} \chi_{R}^{6} d x \\
& \leq\left\|\chi_{R}\right\|_{L^{\infty}}^{4}\left(\int_{\mathbb{R}^{n}}|u|^{2} \chi_{R}^{4} d x\right)^{\frac{1}{2}}\left(\int_{\mathbb{R}^{n}}|\Delta u|^{2} d x\right)^{\frac{1}{2}} \\
& +\frac{6}{R}\left(\int_{\mathbb{R}^{n}}|u|^{2} \chi_{R}^{4} d x\right)^{\frac{1}{2}}\left(\int_{\mathbb{R}^{n}}|\nabla u|^{2^{*}} d x\right)^{\frac{n-2}{2 n}}\left(\int_{\mathbb{R}^{n}}(\nabla \chi)_{R}^{n} \chi_{R}^{3 n} d x\right)^{\frac{1}{n}} \\
& \leq C\left(\|u\|_{\dot{H}^{2}} M\left(u(t), B_{0}(R)\right)^{\frac{1}{2}}\right. \\
& \left.+\frac{1}{R} M\left(u(t), B_{0}(R)\right)^{\frac{1}{2}}\|u\|_{\dot{H}^{2}} R\left\|\left(\nabla \chi^{6}\right)_{R}\right\|_{L^{n}}\right) \\
& \leq C\|u\|_{\dot{H}^{2}} M\left(u(t), B_{0}(R)\right)^{\frac{1}{2}}
\end{aligned}
$$

for some $C>0$ independent of $u$. The second term in the right hand side of (7.4) is even simpler to estimate. We use the Cauchy-Schwartz inequality and (7.2) to get

$$
\begin{aligned}
& \left|\int_{\mathbb{R}^{n}} \Delta u \chi_{R}^{2} \bar{u}\left(\chi_{R}(\Delta \chi)_{R}+3(\nabla \chi)_{R}^{2}\right) d x\right| \\
& \leq C\left(\int_{\mathbb{R}^{n}}|\Delta u|^{2} d x\right)^{\frac{1}{2}}\left(\int_{\mathbb{R}^{n}}|u|^{2} \chi_{R}^{4} d x\right)^{\frac{1}{2}} \\
& \leq C\|u\|_{\dot{H}^{2}} M\left(u(t), B_{0}(R)\right)^{\frac{1}{2}} \\
& \leq C R\|u\|_{\dot{H}^{2}}^{\frac{3}{2}} M\left(u(t), B_{0}(R)\right)^{\frac{1}{4}},
\end{aligned}
$$

where again $C>0$ is independent of $u$. As for the third term in the right hand side of (7.4), we remark that it only has to be considered if $\varepsilon \neq 0$, in which case, $\mathcal{E}$ controls the full norm $H^{2}$, and we estimate this third term by writing that

$$
\begin{aligned}
-2 \varepsilon \operatorname{Re} \int_{\mathbb{R}^{n}} i\left(\frac{4}{R} \bar{u} \chi_{R}^{3} \nabla u(\nabla \chi)_{R}\right) d x & \leq \frac{C}{R}\left(\int_{\mathbb{R}^{n}}|u|^{2} \chi_{R}^{4} d x\right)^{\frac{1}{2}}\left(\int_{\mathbb{R}^{n}}|\nabla u|^{2}\right)^{\frac{1}{2}} \\
& \leq C \frac{\mathcal{E}(u)^{\frac{3}{4}}}{R} M\left(u(t), B_{0}(R)\right)^{\frac{1}{4}}
\end{aligned}
$$

where $C>0$ does not depend on $u$. Finally, putting all these estimates together, we get (7.3). This ends the proof of Lemma 7.1.

Now we discuss localized Morawetz type estimates for (1.2). 
Proposition 7.1. Let $n \geq 5$, and $p=2^{\sharp}-1$. There exists $C>0$ such that

$$
\int_{I} \int_{|x| \leq K|I|^{1 / 4}} \frac{|u(x)|^{2^{\sharp}}}{|x|} d x \leq C\left(K^{3}+K^{-1}\right)\left(\sup _{I} \hat{\mathcal{E}}(u)\right)|I|^{\frac{3}{4}} .
$$

for all $T>0$, all solutions $u \in C\left([0, T], H^{2}\right)$ of $(1.2)$, all $K>0$, and all intervals $I \subset[0, T]$ such that $|I| \leq 1 /(2 K)^{4}$ when $\varepsilon>0$, where $\hat{\mathcal{E}}(u)=\mathcal{E}(u)+\mathcal{E}(u)^{2^{\sharp}} / 2$.

Proof. We fix $u_{0} \in C_{c}^{\infty}\left(\mathbb{R}^{n}\right), h \in C_{c}^{\infty}\left(\mathbb{R}^{n+1}\right)$, and let $v$ solve (3.1). We adopt the convention that repeated indices are summed. Also, for $f, g$ two differentiable functions, we let

$$
\{f, g\}_{p}=\operatorname{Re}(f \nabla \bar{g}-g \nabla \bar{f}) .
$$

Given a smooth compactly supported real valued function $a$, we define the Morawetz action centered at $0, M_{a}^{0}$ by

$$
M_{a}^{0}(t)=2 \int_{\mathbb{R}^{n}} \partial_{j} a(x) \operatorname{Im}\left(\bar{v}(t, x) \partial_{j} v(t, x)\right) d x .
$$

Integrating by parts, straightforward though lengthy computations that we omit here give that

$$
\begin{aligned}
\partial_{t} M_{a}^{0}(t)= & 2 \int_{\mathbb{R}^{n}}\left(2 \partial_{j} v \partial_{k} \bar{v} \partial_{j k} \Delta a-\frac{1}{2}\left(\Delta^{3} a\right)|v|^{2}-4 \partial_{j k} a \partial_{i k} v \partial_{i j} \bar{v}\right. \\
& \left.+\Delta^{2} a|\nabla v|^{2}-\varepsilon\left(2 \partial_{j k} a \partial_{j} v \partial_{k} \bar{v}-\frac{1}{2} \Delta^{2} a|v|^{2}\right)-\partial_{j} a\{h, v\}_{p}^{j}\right) d x .
\end{aligned}
$$

By density, (7.8) remains true when $h \in N(I)$, and $v \in C\left(I, H^{2}\right)$. Now we let $u \in C\left(I, H^{2}\right) \cap M(I)$ be a solution of (1.2) with $p=2^{\sharp}-1$. In particular, $u$ solves (3.1) with

$$
h=\lambda|u|^{2^{\sharp}-2} u, h \in N(I) .
$$

Hence, (7.8) holds true for $u$ with $h$ as above. Besides, using (7.6), we easily remark that

$$
\operatorname{Re} \int_{\mathbb{R}^{n}} \partial_{j} a\{h, u\}_{p}^{j} d x=\frac{4 \lambda}{n} \int_{\mathbb{R}^{n}}(\Delta a)|u|^{2^{\sharp}} d x .
$$

Now we let $a(x)=<x>_{\delta} \chi_{R}(x)$ in (7.8), where

$$
<x>_{\delta}=\left(\delta^{2}+|x|^{2}\right)^{\frac{1}{2}},
$$

$R>0$ is an arbitrary positive real number, and $\chi_{R}$ is as in (7.1). We observe that if $\alpha \in \mathbb{N}^{n}$ is a multi-index, if $R \geq \delta$, and if $R \leq|x| \leq 2 R$, then $\left|D^{\alpha} a(x)\right| \leq C R^{1-|\alpha|}$. 
Consequently, integrating (7.8) over $I$ and using (7.7) and (7.9), we get that

$$
\begin{aligned}
& 2 \int_{I} \int_{|x| \leq R}\left(\frac{4 \sum_{i}\left(\left|\nabla \partial_{i} u\right|^{2}-\left|\partial_{r} \partial_{i} u\right|^{2}\right)}{<x>_{\delta}}+\frac{2(n-1)\left(|\nabla u|^{2}-3\left|\partial_{r} u\right|^{2}\right)}{<x>_{\delta}^{3}}\right) d x \\
& +2 \int_{I} \int_{|x| \leq R}\left(\frac{(n-1)(n-3)|\nabla u|^{2}}{<x>_{\delta}^{3}}+\frac{8 \lambda(n-1)|u|^{2^{\sharp}}}{n<x>_{\delta}}\right) d x+O(\delta) \\
& -\varepsilon \int_{I} \int_{|x| \leq R}\left(\frac{2\left(|\nabla u|^{2}-\left|\partial_{r} u\right|^{2}\right)}{<x>_{\delta}}+\frac{(n-1)(n-3)|u|^{2}}{<x>_{\delta}^{3}}\right) d x \\
& \leq C \int_{I} \int_{R \leq|x| \leq 2 R}\left(R^{-3}|\nabla u|^{2}+R^{-5}|u|^{2}-R^{-1}\left|\nabla^{2} u\right|^{2}+\lambda R^{-1}|u|^{2^{\sharp}}\right) d x \\
& +C \int_{|x| \leq 2 R}[|u \nabla u|]_{t_{1}}^{t_{2}} d x .
\end{aligned}
$$

Letting $\delta \rightarrow 0$ in (7.10) we get that

$$
\begin{aligned}
& 2 \int_{I} \int_{|x| \leq R}\left(\frac{4 \sum_{i}\left(\left|\nabla \partial_{i} u\right|^{2}-\left|\partial_{r} \partial_{i} u\right|^{2}\right)}{|x|}+\frac{2(n-1)\left(|\nabla u|^{2}-3\left|\partial_{r} u\right|^{2}\right)}{|x|^{3}}\right) d x \\
& +2 \int_{I} \int_{|x| \leq R}\left(\frac{(n-1)(n-3)|\nabla u|^{2}}{|x|^{3}}+\frac{8 \lambda(n-1)|u|^{2^{\sharp}}}{n|x|}\right) d x \\
& -\varepsilon \int_{I} \int_{|x| \leq R}\left(\frac{2\left(|\nabla u|^{2}-\left|\partial_{r} u\right|^{2}\right)}{|x|}+\frac{(n-1)(n-3)|u|^{2}}{|x|^{3}}\right) d x \\
& \leq C \int_{I} \int_{R \leq|x| \leq 2 R}\left(R^{-3}|\nabla u|^{2}+R^{-5}|u|^{2}-R^{-1}\left|\nabla^{2} u\right|^{2}+\lambda R^{-1}|u|^{2^{\sharp}}\right) d x \\
& +C \int_{|x| \leq 2 R}[|u \nabla u|]_{t_{1}}^{t_{2}} d x \\
& \leq C|I| R^{-1} \sup _{I}\left(\mathcal{E}(u)+\mathcal{E}(u)^{2^{\sharp} / 2}\right)+C R^{3} \sup _{I} \mathcal{E}(u),
\end{aligned}
$$

where $C$ does not depend on $I, u$, and $R$. The last inequality in (7.11) follows from Hölder's and Sobolev's inequalities and from the fact that, for any $u \in H^{2}$, the $L^{2}$ norm of $\nabla^{2} u$ is bounded by some constant times the $L^{2}$ norm of $\Delta u$. Now we remark that if $u \in H^{2}\left(\mathbb{R}^{n}\right)$ then, as shown in Levandosky and Strauss [20],

$$
\sum_{i}\left(\left|\nabla \partial_{i} u\right|^{2}-\left|\partial_{r} \partial_{i} u\right|^{2}\right) \geq \frac{n-1}{|x|^{2}}\left|\partial_{r} u\right|^{2} .
$$

By (7.12) we see that

$$
\begin{aligned}
& \int_{I} \int_{|x| \leq R} \frac{4}{|x|} \sum_{i}\left(\left|\nabla \partial_{i} u\right|^{2}-\left|\partial_{r} \partial_{i} u\right|^{2}\right) d x d t \\
& +\int_{I} \int_{|x| \leq R} \frac{2(n-1)}{|x|^{3}}\left(|\nabla u|^{2}-3\left|\partial_{r} u\right|^{2}\right) d x d t \geq 0,
\end{aligned}
$$

and when $\varepsilon \leq 0$, letting $R=K|I|^{\frac{1}{4}}$, we get (7.5). Now, we turn to the case $\varepsilon=1$. We assume $R \leq 1 / 2$. The term $\left(|\nabla u|^{2}-\left|\partial_{r} u\right|^{2}\right) /|x|$ in (7.11) is controlled 
by the gradient term $|\nabla u|^{2} /|x|^{3}$. Independently, when $n \geq 5$, integrating by parts $\int Z \cdot \nabla|u|^{2} d x$ for $Z(x)=|x|^{-3} x$, we get that

$$
\int_{\mathbb{R}^{n}} \frac{|u|^{2}}{|x|^{3}} d x \leq\left(\frac{2}{n-3}\right)^{2} \int_{\mathbb{R}^{n}} \frac{\left|\partial_{r} u\right|^{2}}{|x|} d x
$$

Then, using (7.13), we see that when $R=K|I|^{\frac{1}{4}} \leq 1 / 2$, the term $|u|^{2} /|x|^{3}$ in (7.11) is again controlled by the gradient term $|\nabla u|^{2} /|x|^{3}$. As a consequence, (7.5) also holds true when $\varepsilon=1$ if $|I| \leq(2 K)^{4}$.

\section{Global Existence}

We prove Theorem 1.1 in this section. We follow the strategy initiated in Bourgain $[\mathbf{2}]$ and developed in Tao [26]. As in the preceding sections we may assume that $\varepsilon \in\{-1,0,1\}$. We let $H_{\text {rad }}^{2}$ be the subset of $H^{2}$ consisting of radially symmetrical functions. We claim that the following proposition holds true.

Proposition 8.1. Let $n \geq 5$ and $p=2^{\sharp}-1$. Assume $\lambda>0$. Let $u \in$ $C\left(\left[t_{-}, t_{+}\right], H_{\text {rad }}^{2}\right)$ be a radially symmetrical solution of $(1.2)$ such that $\|u\|_{W\left(\left[t_{-}, t_{+}\right]\right)}<$ $\infty$. Then, there exists $K>0$ depending only on $n, \lambda, \mathcal{E}=\sup _{t} \mathcal{E}(u)$, and $\left|t_{+}-t_{-}\right|$ if $\varepsilon>0$, such that

$$
\|u\|_{Z\left(\left[t_{-}, t_{+}\right]\right)} \leq K \text {. }
$$

Besides, in case $\varepsilon \leq 0$, one can take $K=\Lambda(1+\mathcal{E})^{\mathcal{E}^{\Lambda}}$, where $\Lambda>>1$ is a constant depending only on $n$ and $\lambda$.

First we prove that Proposition 8.1 implies Theorem 1.1. Then we prove the proposition.

Proof of Theorem 1.1. By Corollary 4.1 in Section 4 me may assume that $n \geq 5$ and $p=2^{\sharp}-1$. Let $u_{0} \in H_{\text {rad }}^{2}$ be radially symmetrical. By the Strichartz estimates (3.10), there exists $T>0$ such that (5.2) holds true for $I=[0, T]$. Then Proposition 5.1 gives that there exists $u \in C\left(I, H^{2}\right)$ which solves (1.2) with $p=$ $2^{\sharp}-1$ and such that $u(0)=u_{0}$. Proposition 5.2 allows us to extend $u$ on a maximal interval $\left[0, T^{\star}\right)$ such that $u \in M\left(I^{\prime}\right)$ for any compact subinterval $I^{\prime} \subset\left[0, T^{\star}\right)$, and such that if $T^{\star}<+\infty$, then the $Z\left(\left[0, T^{\star}\right]\right)$-norm of $u$ is infinite. Besides, it follows from uniqueness that $u$ is spherically symmetrical. Now suppose by contradiction that $T^{\star}<+\infty$, and let $I^{\prime} \subset\left[0, T^{\star}\right)$ be a compact subinterval of $\left[0, T^{\star}\right)$. By Proposition 8.1, the $Z\left(I^{\prime}\right)$-norm of $u$ is bounded by some finite quantity independent of the subinterval $I^{\prime}$. Since this contradicts the fact that $u$ must blow-up in the $Z$-norm, we get that $T^{\star}=+\infty$ and that $u$ is a global solution of (1.2). This proves Theorem 1.1.

As a remark the bound (8.1) has interest in its own. It is of importance when discussing scattering as in Section 9 below. Now we prove Proposition 8.1 and split the proof into several steps.

STEP 8.1. Let $u \in C\left(\left[t_{1}, t_{2}\right], H_{\text {rad }}^{2}\right)$ be a radially symmetrical solution of (5.1) on $I=\left[t_{1}, t_{2}\right]$. If $\varepsilon=1$, assume also that $|I| \leq 1$. There exists $\eta_{0}>0$ depending only on $n$ and $\lambda$ such that if

$$
\frac{1}{4} \eta \leq\|u\|_{W(I)} \leq \eta
$$


for some $0<\eta \leq \eta_{0}$, then

$$
\left\|u_{k}\right\|_{W(I)} \geq \frac{1}{8} \eta
$$

where $u_{k}=e^{i\left(t-t_{k}\right)\left(\Delta^{2}+\varepsilon \Delta\right)} u\left(t_{k}\right)$ for $k=1,2$.

Proof OF SteP 8.1. We prove this for $u_{1}$, the proof for $u_{2}$ being similar. Using Duhamel's formula and (3.19), we write

$$
\left\|u_{k}\right\|_{W(I)} \geq\|u\|_{W(I)}-C\left\||u|^{\frac{8}{n-4}} u\right\|_{N(I)} \geq \frac{1}{4} \eta-C \eta^{\frac{n+4}{n-4}} .
$$

Noting that (8.4) gives (8.3) provided that $\eta \leq \eta_{0}$ is sufficiently small, this proves Step 8.1.

From now on we consider a radially symmetrical solution $u \in C\left(\left[t_{-}, t_{+}\right], H_{\text {rad }}^{2}\right)$ of (5.1) with $\lambda>0$. Besides, in case $\varepsilon=1$, we also assume that $\left|t_{+}-t_{-}\right| \leq 1$. By energy and mass conservation, we have

$$
\mathcal{E}=\sup _{t} \mathcal{E}(u(t)) \leq E\left(u_{0}\right)+M\left(u_{0}\right) .
$$

Moreover, by Proposition 5.1 and the Strichartz estimates (3.10), we know that there exists $\epsilon_{0}>0$ such that (8.1) holds true if there exists a time $t$ such that $\mathcal{E}(u(t))<\epsilon_{0}$. Without loss of generality we may then assume that the energy is not too small in the sense that, for any $t \in\left[t_{-}, t_{+}\right], \mathcal{E}(u(t)) \geq \epsilon_{0}$ for some $\epsilon_{0}>0$. Let $\eta>0$ be small. We split $\left[t_{-}, t_{+}\right]$into $N$ disjoint intervals, $\left(I_{j}\right)_{1 \leq j \leq N}$ such that (8.2) holds true on each interval. We let

$$
u_{ \pm}(t)=e^{i\left(t-t_{ \pm}\right)\left(\Delta^{2}+\varepsilon \Delta\right)} u\left(t_{ \pm}\right),
$$

and, following the terminology in Tao $[\mathbf{2 6}]$, we call an interval $I_{j}$ exceptional if one of the following conditions holds true:

$$
\begin{aligned}
& \left\|u_{+}\right\|_{W\left(I_{j}\right)}>\eta^{K_{2}}, \text { or } \\
& \left\|u_{-}\right\|_{W\left(I_{j}\right)}>\eta^{K_{2}},
\end{aligned}
$$

where $K_{2}=24 n^{2}$. An interval is said to be unexceptional if it is not exceptional. Using the Strichartz estimates (3.10) and Sobolev's inequality, we get an upper bound for the number $N_{e}$ of exceptional intervals. Namely

$$
N_{e} \leq C\left(\left\|u_{0}\right\|_{\dot{H}^{2}} \eta^{-K_{2}}\right)^{\frac{2(n+4)}{n-4}}+1 .
$$

If all intervals are exceptional, (8.2) and (8.6) give the bound (8.1), and this proves the proposition. From now on we assume that there exist unexceptional intervals. A consequence of Lemma 8.1 is that the extremal intervals $I_{1}$ and $I_{N}$ are always exceptional, provided that $\eta$ is sufficiently small. The next step exhibits concentration when dealing with unexceptional intervals.

STEP 8.2. Let $u \in C\left(\left[t_{-}, t_{+}\right], H_{\text {rad }}^{2}\right)$ be a radially symmetrical solution of (5.1) and let $I=\left[t_{0}, t_{1}\right]$ be an unexceptional interval for $u$ such that $|I| \leq 1$ if $\varepsilon=1$. Then there exists $x_{0} \in \mathbb{R}^{n}$ such that for any $t \in I$,

$$
M\left(u(t), B_{x_{0}}\left(2 \eta^{-K_{1}}|I|^{\frac{1}{4}}\right)\right) \geq C \eta^{K_{1}} \mathcal{E}^{-\frac{n+2}{2}}|I|,
$$

where $K_{1}=n^{2}+6 n+4, C>0$ is independent of $I, x_{0}$ and $u$, and we assume that $\eta$ is sufficiently small in the sense that $\eta<\mathcal{E}^{-5 n} \eta_{1}$ for some $\eta_{1}>0$ depending only on $n$ and $\lambda$. 
Proof of Step 8.2. We consider $I^{1}=\left[t_{0}, \frac{t_{0}+t_{1}}{2}\right]$ and $I^{2}=\left[\frac{t_{0}+t_{1}}{2}, t_{1}\right]$. By time reversal and time translation symmetries, and by (8.2), we can assume that

$$
\|u\|_{W\left(I^{2}\right)} \geq \frac{1}{4} \eta
$$

Besides, by Duhamel's formula, we get that for any $t \in I^{2}$,

$$
\begin{aligned}
u(t)= & u_{-}(t)+i \lambda \int_{t_{-}}^{t_{0}} e^{i(t-s)\left(\Delta^{2}+\varepsilon \Delta\right)}|u(s)|^{\frac{8}{n-4}} u(s) d s \\
& +i \lambda \int_{t_{0}}^{t} e^{i(t-s)\left(\Delta^{2}+\varepsilon \Delta\right)}|u(s)|^{\frac{8}{n-4}} u(s) d s .
\end{aligned}
$$

Since $I$ is unexceptional, the first term in the right hand side of (8.9) is small in the $W$-Norm. As for the third term in the righthand side of (8.9), we use Sobolev's inequality and the Strichartz estimates (3.19) to write that

$$
\begin{aligned}
& \left\|\int_{t_{0}}^{t} e^{i(t-s)\left(\Delta^{2}+\varepsilon \Delta\right)}|u(s)|^{\frac{8}{n-4}} u(s) d s\right\|_{W(I)} \\
& \leq C\left\|\int_{t_{0}}^{t} e^{i(t-s)\left(\Delta^{2}+\varepsilon \Delta\right)}|u(s)|^{\frac{8}{n-4}} u(s) d s\right\|_{M(I)} \\
& \leq C\left\||u|^{\frac{8}{n-4}} u\right\|_{N(I)} \leq C\|u\|_{W(I)}^{\frac{n+4}{n-4}} \leq C \eta^{\frac{n+4}{n-4}}
\end{aligned}
$$

where $C>0$ depends only on $n$. Hence, if we define $v(t)$ for $t \in I$ by

$$
v(t)=\int_{t_{-}}^{t_{0}} e^{i(t-s)\left(\Delta^{2}+\varepsilon \Delta\right)}|u(s)|^{\frac{8}{n-4}} u(s) d s,
$$

we get from (8.5) and (8.8)-(8.11) that, if $\eta$ is sufficiently small, then

$$
\|v\|_{W\left(I^{2}\right)} \geq \frac{1}{4} \eta-\eta^{K_{2}}-C \eta^{\frac{n+4}{n-4}} \geq \frac{1}{8} \eta .
$$

For later use, we remark that, by $(8.11), v$ satisfies the linear equation

$$
i \partial_{t} v+\Delta^{2} v+\varepsilon \Delta v=0
$$

and we get that

$$
\|v\|_{L^{\infty}\left(I, \dot{H}^{2}\right)} \leq\left\|v\left(t_{0}\right)\right\|_{\dot{H}^{2}} \leq \lambda^{-1}\left(\left\|u\left(t_{0}\right)\right\|_{\dot{H}^{2}}+\left\|u\left(t_{-}\right)\right\|_{\dot{H}^{2}}\right) \leq 2 \lambda^{-1} \mathcal{E}^{\frac{1}{2}} .
$$

Besides, the Strichartz estimates (3.19) give that

$$
\begin{aligned}
& \|u\|_{M(I)} \leq C\left\|u\left(t_{0}\right)\right\|_{\dot{H}^{2}}+C\left\||u|^{\frac{8}{n-4}} u\right\|_{N(I)} \leq C \mathcal{E}^{\frac{1}{2}}+C \eta^{\frac{n+4}{n-4}}, \text { and } \\
& \left\|u_{-}\right\|_{M(I)} \leq C\left\|u\left(t_{-}\right)\right\|_{\dot{H}^{2}} \leq C \mathcal{E}^{\frac{1}{2}}
\end{aligned}
$$

where $C>0$ does not depend on $u$ and $I$. Then (8.9), (8.10), and (8.15) give

$$
\|v\|_{M\left(I^{2}\right)} \leq C \mathcal{E}^{\frac{1}{2}}+C \mathcal{E}^{\frac{1}{2}}+2 C \eta^{\frac{n+4}{n-4}} \leq 3 C \mathcal{E}^{\frac{1}{2}}
$$

Independently, integration by parts and boundedness of Riesz transforms give that there exists $C>0$ independent of $I$ such that for any function $g \in M(I)$,

$$
\|g\|_{W(I)} \leq C\|g\|_{M(I)}^{\frac{1}{2}}\|g\|_{Z(I)}^{\frac{1}{2}} .
$$

Then, (8.12), (8.16), and (8.17) give that

$$
\|v\|_{Z\left(I^{2}\right)} \geq C \eta^{2} \mathcal{E}^{-\frac{1}{2}}
$$


where $C>0$ depends only on $n$ and $\lambda$. Now, we prove that $v$ enjoys additional regularity. Let us define

$$
v_{a v}(t, x)=\frac{1}{V} \int_{B_{0}(2)} v(t, x-r y) \chi(y) d y,
$$

where $\chi$ is a bump function as in (7.1), r $=\eta^{n+5}|I|^{\frac{1}{4}}$, and $V=\int \chi d x$. We claim that

$$
\left\|v-v_{a v}\right\|_{Z\left(I^{2}\right)} \leq C \mathcal{E}^{\frac{n+4}{2(n-4)}} \eta^{\frac{2 n+10}{n+4}} .
$$

Now we prove (8.20). For $k \in \mathbb{R}^{n}$, we let $\tau_{k}$ be defined on a function $g$ by $\tau_{k} g(x)=$ $g(x+k)$. We first assume that $5 \leq n \leq 12$. Then, by (3.7) and Hölder's inequality, letting $f(u)=|u|^{\frac{8}{n-4}} u$, we get that

$$
\begin{aligned}
& \left\|v-\tau_{k} v\right\|_{L^{\infty}\left(I^{2}, L^{\frac{2(n+4)}{n-4}}\right)} \\
& \leq \sup _{t \in I^{2}}\left\|\int_{t_{-}}^{t_{0}} e^{i(t-s)\left(\Delta^{2}+\varepsilon \Delta\right)}\left(f(u(s))-f\left(\tau_{k} u(s)\right)\right) d s\right\|_{L^{\frac{2(n+4)}{n-4}}} \\
& \leq C \sup _{t \in I^{2}} \int_{t_{-}}^{t_{0}}|t-s|^{-\frac{2 n}{n+4}}\left\|f(u(s))-f\left(\tau_{k} u(s)\right)\right\|_{L^{\frac{2(n+4)}{n+12}}} d s \\
& \leq C|I|^{-\frac{n-4}{n+4}} \|\left.\left(|u|^{\frac{8}{n-4}}+\left|\tau_{k} u\right|^{\frac{8}{n-4}}\right)\left|u-\tau_{k} u\right|\right|_{L^{\infty} L^{\frac{2(n+4)}{n+12}}} \\
& \leq C|I|^{-\frac{n-4}{n+4}}\|u\|_{L^{\infty} L^{2^{\sharp}}}^{\frac{8}{n-4}}\left\|u-\tau_{k} u\right\|_{L^{\infty} L^{2^{\sharp}}}^{\frac{12-n}{n+4}}\left\|u-\tau_{k} u\right\|_{L^{\infty} L^{\frac{2 n}{n-2}}}^{\frac{2(n-4)}{n+4}} \\
& \leq C|I|^{-\frac{n-4}{n+4}} \mathcal{E}^{\frac{-n^{2}+24 n-16}{2\left(n^{2}-16\right)}}\left\|u-\tau_{k} u\right\|_{L^{\infty} L^{\frac{2 n}{n-2}}}^{\frac{2(n-4)}{n+4}} .
\end{aligned}
$$

By conservation of energy and Sobolev's inequality,

$$
\left\|u-\tau_{k} u\right\|_{L^{\infty}\left(I, L^{\frac{2 n}{n-2}}\right)} \leq|k|\|\nabla u\|_{L^{\infty}\left(I, L^{\frac{2 n}{n-2}}\right)} \leq C|k| \mathcal{E}^{\frac{1}{2}} .
$$

Combining (8.19), (8.21) and the above computation, we get with Hölder's inequality that

$$
\begin{aligned}
\left\|v-v_{a v}\right\|_{Z\left(I^{2}\right)} & \leq \frac{1}{V} \int_{B_{0}(2)} \chi(y)\left\|v-\tau_{r y} v\right\|_{Z\left(I^{2}\right)} d y \\
& \leq C|I|^{\frac{n-4}{2(n+4)}} \int_{B_{0}(2)} \chi(y)\left\|v-\tau_{r y} v\right\|_{L^{\infty}\left(I^{2}, L^{\frac{2(n+4)}{n-4}}\right)} d y \\
& \leq C\left(r|I|^{-\frac{1}{4}}\right)^{\frac{2(n-4)}{n+4}} \mathcal{E}^{\frac{n+4}{2(n-4)}} .
\end{aligned}
$$

Since $\eta<1$, (8.22) gives (8.20) when $5 \leq n \leq 12$. When $n \geq 13$, we first estimate the gradient of $v$ with (3.7). We have that

$$
\begin{aligned}
\|\nabla v\|_{L^{\infty}\left(I^{2}, L^{\left.\frac{2 n}{n-6}\right)}\right.} & \leq \int_{t_{-}}^{t_{0}}\left\|\nabla e^{i(t-s)\left(\Delta^{2}+\varepsilon \Delta\right)} f(u(s))\right\|_{L^{\infty}\left(\left[t_{-}, t_{0}\right], L^{\frac{2 n}{n-6}}\right)} d s \\
& \leq C \int_{t_{-}}^{t_{0}}|t-s|^{-\frac{3}{2}}\|\nabla f(u)\|_{L^{\infty}\left(\left[t_{-}, t_{0}\right], L^{\left.\frac{2 n}{n+6}\right)}\right.} d s \\
& \leq C|I|^{-\frac{1}{2}}\left\||u|^{\frac{8}{n-4}}\right\|_{L^{\infty}\left(\left[t_{-}, t_{0}\right], L^{\frac{n}{4}}\right)}\|\nabla u\|_{L^{\infty}\left(\left[t_{-}, t_{0}\right], L^{\frac{2 n}{n-2}}\right)} \\
& \leq C|I|^{-\frac{1}{2}} \mathcal{E}^{\frac{n+4}{2(n-4)}} .
\end{aligned}
$$


Besides, Sobolev's inequality and (8.14) imply that $\|\nabla v\|_{L^{\infty}\left(I^{2}, L^{\frac{2 n}{n-2}}\right)} \leq C \mathcal{E}^{\frac{1}{2}}$, and by Hölder's inequality, this gives

$$
\|\nabla v\|_{L^{\infty}\left(I^{2}, L^{2 \sharp}\right)} \leq C \mathcal{E}^{\frac{n}{2(n-4)}}|I|^{-\frac{1}{4}} .
$$

Then, an application of Sobolev's inequality with (8.23) and (8.24) yields

$$
\begin{aligned}
\left\|v-\tau_{k} v\right\|_{L^{\infty}\left(I^{2}, L^{\frac{2(n+4)}{n-4}}\right)} & \leq\left\|v-\tau_{k} v\right\|_{L^{\infty}\left(I^{2}, L^{\frac{2 n}{n-8}}\right)}^{\frac{n-4}{n+4}}\left\|v-\tau_{k} v\right\|_{L^{\infty}\left(I^{2}, L^{2}\right)}^{\frac{8}{n+4}} \\
& \leq C|I|^{-\frac{n-4}{2(n+4)} \mathcal{E}^{\frac{n^{2}+8 n-16}{2\left(n^{2}-16\right)}}}\left(|k||I|^{-\frac{1}{4}}\right)^{\frac{8}{n+4}} .
\end{aligned}
$$

Then, by proceeding as in (8.22), we finally find

$$
\left\|v-v_{a v}\right\|_{Z\left(I^{2}\right)} \leq C \mathcal{E}^{\frac{n^{2}+8 n-16}{2\left(n^{2}-16\right)}}\left(r|I|^{-\frac{1}{4}}\right)^{\frac{8}{n+4}} .
$$

Combining (8.22) and (8.25), we get (8.20) for all $n \geq 5$. Now by (8.18) and (8.20), we get that if $\eta$ is sufficiently small, namely $\eta<C \mathcal{E}^{-5 n}$, then

$$
\left\|v_{a v}\right\|_{Z\left(I^{2}\right)} \geq C \eta^{2} \mathcal{E}^{-\frac{1}{2}} \text {. }
$$

Independently, (8.14) and Sobolev's inequality give the bound

$$
\left\|v_{a v}\right\|_{L^{2^{\sharp}\left(I^{2} \times \mathbb{R}^{n}\right)}} \leq C|I|^{\frac{n-4}{2 n}} \mathcal{E}^{\frac{1}{2}} .
$$

An application of Hölder's inequality with (8.26) and (8.27) then gives

$$
\left\|v_{a v}\right\|_{L^{\infty}\left(I^{2} \times \mathbb{R}^{n}\right)} \geq C \eta^{\frac{n+4}{2}}|I|^{-\frac{n-4}{8}} \mathcal{E}^{-\frac{n+2}{4}},
$$

and we obtain with (8.28) that there exists a point $\left(t_{0}, x_{0}\right) \in I^{2} \times \mathbb{R}^{n}$ such that

$$
\left|\int_{B_{0}(2)} \chi(y) v\left(t_{0}, x_{0}-r y\right) d y\right| \geq \frac{1}{2} C \eta^{\frac{n+4}{2}}|I|^{-\frac{n-4}{8}} \mathcal{E}^{-\frac{n+2}{4}} .
$$

It follows from (8.29) and Hölder's inequality that

$$
M\left(v\left(t_{0}\right), B_{x_{0}}(2 r)\right) \geq C \eta^{K_{1}}|I| \mathcal{E}^{-\frac{n+2}{2}},
$$

where $K_{1}=n^{2}+6 n+4$. Now, since $v$ satisfies (8.13), using (7.3), we get that, for any $t \in I$,

$$
\partial_{t}\left(M\left(\left(v(t), B_{x_{0}}\left(2 \eta^{-K_{1}}|I|^{\frac{1}{4}}\right)\right)\right)^{\frac{3}{4}} \leq C \mathcal{E}^{\frac{3}{4}} \eta^{K_{1}}|I|^{-\frac{1}{4}} .\right.
$$

Integrating (8.31) over $I$, using (8.30), we get that for any $t \in I$,

$$
M\left(v(t), B_{x_{0}}\left(2 \eta^{-K_{1}}|I|^{\frac{1}{4}}\right)\right) \geq C \eta^{K_{1}}|I| \mathcal{E}^{-\frac{n+2}{2}} .
$$

In particular, (8.32) holds true for $t=t_{0}$. Independently, since $I$ is unexceptional, by (8.5) we can find some time $\tau \in I$ such that

$$
\left\|u_{-}(\tau)\right\|_{L^{\frac{2(n+4)}{n-4}\left(\mathbb{R}^{n}\right)}} \leq C \eta^{K_{2}}|I|^{-\frac{n-4}{2(n+4)}} .
$$

Then, (8.33) and Hölder's inequality gives

$$
M\left(u_{-}(\tau), B_{x_{0}}\left(2 \eta^{-K_{1}}|I|^{\frac{1}{4}}\right)\right) \leq C \eta^{8 K_{1}}|I|
$$

and, again, since $u_{-}$satisfies (3.1) with $h=0$, we get with (8.34) that for any $t \in I$

$$
M\left(u_{-}(t), B_{x_{0}}\left(2 \eta^{-K_{1}}|I|^{\frac{1}{4}}\right)\right) \leq C|I| \eta^{\frac{4}{3} K_{1}} \mathcal{E} \text {. }
$$


Now, by (8.9), (8.11), and estimates (8.30) and (8.35), we get that, at time $t_{0}$,

$$
M\left(u\left(t_{0}\right), B_{x_{0}}\left(2 \eta^{-K_{1}}|I|^{\frac{1}{4}}\right)\right) \geq C|I| \eta^{K_{1}} \mathcal{E}^{-\frac{n+2}{2}} .
$$

A final application of (7.3), using (8.36), gives (8.7). This ends the proof of Step 8.2 .

A consequence of Step 8.2 is as follows.

STEP 8.3. Let $u \in C\left(\left[t_{-}, t_{+}\right], H_{\text {rad }}^{2}\right)$ be a radially symmetrical solution of (5.1) and let $I$ be an unexceptional interval such that $|I| \leq 1$ if $\varepsilon=1$. Then

$$
\int_{I} \int_{B_{0}\left(2 \eta^{\left.-4 K_{1}|I|^{\frac{1}{4}}\right)}\right.} \frac{|u(t, x)|^{\frac{2 n}{n-4}}}{|x|} d x \geq C \eta^{13 K_{1}} \mathcal{E}^{-4 n}|I|^{\frac{3}{4}},
$$

where $K_{1}=n^{2}+6 n+4$, and $C>0$ is a constant depending only on $n$ and $\lambda$.

Proof Of SteP 8.3. By Hölder's inequality and (8.7) the following bound from below holds true. Namely, that for any $t \in I$,

$$
\int_{B_{x_{0}}\left(2 \eta^{\left.-K_{1}|I|^{\frac{1}{4}}\right)}\right.}|u(t, x)|^{2^{\sharp}} d x \geq C \eta^{9 K_{1}} \mathcal{E}^{-4 n} .
$$

Now we claim that $\left|x_{0}\right| \leq \eta^{-4 K_{1}}|I|^{\frac{1}{4}}$. Indeed, if this is not the case, then there exists at least $\eta^{-3(n-1) K_{1}} / 4^{n-1}$ disjoint balls which can be obtained by rotating $B_{x_{0}}\left(2 \eta^{-K_{1}}\right)$. Using the radial symmetry assumption and (8.38) we get that, for any $t \in I$,

$$
2^{\sharp} E(u(t)) \geq\|u(t)\|_{L^{2 \sharp}}^{2^{\sharp}} \geq \frac{1}{4} \eta^{-3(n-1) K_{1}} C \eta^{9 K_{1}} \mathcal{E}^{-4 n} \geq C \eta^{-2 K_{1}} \mathcal{E}^{-4 n},
$$

and (8.39) contradicts $E(u(t)) \leq \mathcal{E}$ if $\eta$ is sufficiently small. Then, by (8.7) we get that for any $t \in I$,

$$
M\left(u(t), B_{0}\left(2 \eta^{-4 K_{1}}|I|^{\frac{1}{4}}\right)\right) \geq C \eta^{K_{1}} \mathcal{E}^{-\frac{n+2}{2}}|I|
$$

provided that $\eta<\mathcal{E}^{-5 n} \eta_{1}$ where $\eta_{1}$ is sufficiently small depending only on $n$ and $\lambda$. Using Hölder's inequality and (8.40), we obtain (8.37).

The bound from below in Step 8.3 can be combined with the bound stemming from the localized Morawetz estimate (7.5), and we then get that the following holds true.

STEP 8.4. Let $u \in C\left(\left[t_{-}, t_{+}\right], H_{\text {rad }}^{2}\right)$ be a radially symmetrical solution of (5.1) and let $I=\bigcup_{j_{1} \leq j \leq j_{2}} I_{j}$ be a collection of consecutive unexceptional intervals for $u$. In case $\varepsilon=1$, suppose that $|I| \leq \eta^{16 K_{1}} / 256$. Then there exists $j_{1} \leq j_{0} \leq j_{2}$ such that

$$
\left|I_{j_{0}}\right| \geq K|I|,
$$

where $K=C \eta^{100 K_{1}} \mathcal{E}^{-20 n}$, and $C>0$ is a constant depending only on $n$ and $\lambda$. 
Proof of SteP 8.4. Estimates (8.37) and (7.5) give that

$$
\begin{aligned}
C \eta^{13 K_{1}} \mathcal{E}^{-4 n} \sum_{j_{1} \leq j \leq j_{2}}\left|I_{j}\right|^{\frac{3}{4}} & \leq \sum_{j} \int_{I_{j}} \int_{B_{0}\left(2 \eta^{\left.-4 K_{1}\left|I_{j}\right|^{\frac{1}{4}}\right)}\right.} \frac{|u|^{2^{\sharp}}}{|x|} d x \\
& \leq \int_{I} \int_{B_{0}\left(2 \eta^{-4 K_{1}|I|^{\frac{1}{4}}}\right)} \frac{|u|^{\sharp}}{|x|} d x \\
& \leq C \eta^{-12 K_{1}}\left(\mathcal{E}+\mathcal{E}^{\frac{n}{n-4}}\right)|I|^{\frac{3}{4}} .
\end{aligned}
$$

Let $\tilde{K}=C \mathcal{E}^{5 n} \eta^{-25 K_{1}}$. We get from (8.42) that

$$
\left(\max _{j}\left|I_{j}\right|\right)^{-\frac{1}{4}} \sum_{j}\left|I_{j}\right| \leq \sum_{j}\left|I_{j}\right|^{\frac{3}{4}} \leq \tilde{K}|I|^{\frac{3}{4}} \leq \tilde{K}\left(\sum_{j}\left|I_{j}\right|\right)^{\frac{3}{4}}
$$

and Step 8.4 easily follows from (8.43).

At this point we need a combinatorial argument. Such a result goes back to Bourgain $[\mathbf{2}]$ and Tao $[\mathbf{2 6}]$. In the form we use it here, the proposition is due to Killip, Visan, and Zhang [18].

Proposition 8.2. Let I be an interval tiled by finitely many intervals $I_{1}, \ldots, I_{N}$. Suppose that for any contiguous family $\left\{I_{j}: j \in \mathcal{J}\right\}$ there exists $j_{*} \in \mathcal{J}$ so that

$$
\left|I_{j_{*}}\right| \geq K\left|\cup_{j \in \mathcal{J}} I_{j}\right|
$$

for some $K>0$. Then there exists $M \geq \ln (N) / \ln \left(2 K^{-1}\right)$, and distinct indices $j_{1}, \ldots, j_{M}$, such that

$$
\begin{aligned}
& \left|I_{j_{1}}\right| \geq 2\left|I_{j_{2}}\right| \geq \cdots \geq 2^{M-1}\left|I_{j_{M}}\right|, \text { and } \\
& \operatorname{dist}\left(I_{j_{l}}, I_{j_{k}}\right) \leq(2 K)^{-1}\left|I_{j_{l}}\right|
\end{aligned}
$$

for all $l<k$.

At last we need the following step.

STEP 8.5. Let $u \in C\left(\left[t_{-}, t_{+}\right], H_{\text {rad }}^{2}\right)$ be a radially symmetrical solution of (5.1) and let $I_{j_{1}}, \ldots, I_{j_{M}}$ be a disjoint family of unexceptional intervals for $u$ obeying (8.44) with $K=C \eta^{100 K_{1}} \mathcal{E}^{-20 n}$. In case $\varepsilon=1$, suppose also that $\left|I_{j_{1}}\right| \leq 1$. Then $M \leq C \mathcal{E} \eta^{-5000 n^{2}} \ln (1 / \eta)$, where $C$ depends only on $n$ and $\lambda$.

Proof of Proposition 8.5. We let $t_{*} \in I_{j_{M}}$. We can combine (8.40) with (7.3) and (8.44) to get that for any $1 \leq k \leq M$, the following mass concentration estimate holds true. Namely that

$$
M\left(u\left(t_{*}\right), B_{0}\left(2 \eta^{-101 K_{1}}\left|I_{j_{k}}\right|^{\frac{1}{4}}\right)\right) \geq C \eta^{K_{1}} \mathcal{E}^{-\frac{n+2}{2}}\left|I_{j_{k}}\right|
$$

provided that $\eta<C \mathcal{E}^{-21 n}$. Besides, (7.2) also gives that

$$
M\left(u\left(t_{*}\right), B_{0}(R)\right) \leq C \mathcal{E} R^{4} .
$$

Let us consider the family of annuli

$$
A_{k}=\left\{x: \eta^{K_{1}}\left|I_{j_{k}}\right|^{\frac{1}{4}} \leq|x| \leq 2 \eta^{-101 K_{1}}\left|I_{j_{k}}\right|^{\frac{1}{4}}\right\} .
$$


Then (8.45) and (8.46) give the following bound from below for the mass in $A_{k}$. Namely that

$$
\int_{A_{k}}\left|u\left(t_{*}, x\right)\right|^{2} d x \geq C \eta^{K_{1}} \mathcal{E}^{-\frac{n+2}{2}}\left|I_{j_{k}}\right|,
$$

and with Hölder's inequality we deduce from (8.47) that

$$
\int_{A_{k}}\left|u\left(t_{*}, x\right)\right|^{2^{\sharp}} d x \geq C \eta^{K_{3}},
$$

where $K_{3}=5\left(10^{3}\right) n^{2}$. Now, it only remains to observe that, thanks to (8.44), if $l=405 K_{1} \ln \left(\frac{1}{\eta}\right) / \ln 2$, then the annuli $A_{j_{1+k l}}$ are disjoint for all $k$. By conservation of energy and (8.48) it follows that there are at most $C \mathcal{E} \eta^{-K_{3}}$ such annuli, and this proves Step 8.5.

Thanks to Steps 8.1 to 8.5 we are now in position to prove Proposition 8.1.

Proof of Proposition 8.1. Suppose first that $\varepsilon \leq 0$. As mentioned before, see (8.6), it is easy to bound the number $N_{e}$ of exceptional intervals. Now, consider a gap $J=\cup_{j \in \mathcal{J}} I_{j}$ between two exceptional intervals. The gap $J$ is made exclusively of unexceptional intervals $I_{j}$ and then, applying (8.41), (8.44), and Step 8.5, we see that

$$
|\mathcal{J}| \leq C \eta^{-C \eta^{-5000 n^{2}}}
$$

if $\eta \leq \eta_{1} \mathcal{E}^{-5 n}$ for $\eta_{1}$ sufficiently small depending only on $n$ and $\lambda$. Since there are at most $N_{e}$ such gaps, we get the desired conclusion. In case $\varepsilon=1$, we first split $\left[t_{-}, t_{+}\right]$into subintervals $\tilde{I}_{k \in \mathcal{K}}$ such that $\left|\tilde{I}_{k}\right| \leq 1 / 256 \eta^{48 n^{2}}$. Then, for any $k$, we can apply on $I_{k}$ the same strategy as in the case $\varepsilon \leq 0$, and we see that the $Z\left(\tilde{I}_{k}\right)$-norm of $u$ is bounded by a constant $C(\mathcal{E}, \eta, n)$. Then, since there are at most $C \eta^{-48 n^{2}}\left|t_{+}-t_{-}\right|+1$ such intervals, we also get Proposition 8.1 when $\varepsilon=1$.

\section{Scattering for the critical equation}

We briefly discuss scattering in this section and prove that, by standard procedures, an estimate like (8.1) implies scattering when $\varepsilon \leq 0$. By scaling invariance we may assume $\varepsilon=-1$ or $\varepsilon=0$. In proposition 9.1 we prove that solutions of (1.2) with $p=2^{\sharp}-1$ and $\lambda>0$ converge to a scattering state. We construct the scattering operator in Proposition 9.2.

Proposition 9.1. Let $n \geq 5$. Given any $u \in C\left(\mathbb{R}, H_{\text {rad }}^{2}\right)$ a radially symmetrical solution of (1.2) with $p=2^{\sharp}-1, \lambda>0$, and $\varepsilon \leq 0$, there exists $u^{ \pm} \in H_{\text {rad }}^{2}$ such that

$$
\left\|u(t)-e^{i t\left(\Delta^{2}+\varepsilon \Delta\right)} u^{ \pm}\right\|_{H^{2}} \rightarrow 0
$$

as $t \rightarrow \pm \infty$. The functions $u^{ \pm}$are unique, they are determined by (9.1), and we have that

$$
\begin{aligned}
M\left(u_{0}\right) & =M\left(u^{ \pm}\right), \text {and } \\
2 E\left(u_{0}\right) & =\left\|u^{ \pm}\right\|_{\dot{H}^{2}}^{2}-\varepsilon\left\|u^{ \pm}\right\|_{\dot{H}^{1}}^{2} .
\end{aligned}
$$

This defines two mappings $S_{ \pm}: u(0) \mapsto u^{ \pm}$from $H_{\text {rad }}^{2}$ into $H_{\text {rad }}^{2}$, and $S_{+}$and $S_{-}$ are continuous in $\mathrm{H}_{\text {rad }}^{2}$. 
Proof. By time reversal symmetry it suffices to prove (9.1) for $u^{+}$. From Proposition 8.1, we see that $u$ has bounded $Z$-norm over $\mathbb{R}_{+}$, and from Proposition 5.2 , this provides an a priori bound for the $W\left(\mathbb{R}_{+}\right)$-norm of $u$. Independently, Since $e^{i t\left(\Delta^{2}+\varepsilon \Delta\right)}$ is an isometry on $H^{2},(9.1)$ is equivalent to proving that there exists $u^{+} \in H^{2}$ such that

$$
\left\|e^{-i t\left(\Delta^{2}+\varepsilon \Delta\right)} u(t)-u^{+}\right\|_{H^{2}} \rightarrow 0
$$

as $t \rightarrow+\infty$. Now we prove that $e^{-i t\left(\Delta^{2}+\varepsilon \Delta\right)} u(t)$ satisfies a Cauchy criterion. Duhamel's formula gives that

$$
e^{-i t_{1}\left(\Delta^{2}+\varepsilon \Delta\right)} u\left(t_{1}\right)-e^{-i t_{0}\left(\Delta^{2}+\varepsilon \Delta\right)} u\left(t_{0}\right)=i \lambda \int_{t_{0}}^{t_{1}} e^{-i s\left(\Delta^{2}+\varepsilon \Delta\right)}|u(s)|^{\frac{8}{n-4}} u(s) d s .
$$

By (3.20) with $(a, b)=(2,2 n /(n-2))$, we see from the finiteness of the $W\left(\mathbb{R}_{+}\right)$norm of $u$ that the righthand side in (9.4) is like $o(1)$ in $\dot{H}^{2}$ as $t_{0}, t_{1} \rightarrow+\infty$. In particular, $e^{-i t\left(\Delta^{2}+\varepsilon \Delta\right)} u(t)$ satisfies a Cauchy criterion, and there exists $u^{+} \in H^{2}$ such that (9.3) holds true. We also get that

$$
u^{+}=u_{0}+i \lambda \int_{0}^{\infty} e^{-i s\left(\Delta^{2}+\varepsilon \Delta\right)}|u(s)|^{\frac{8}{n-4}} u(s) d s,
$$

and $u^{+}$is unique. Let us now prove that the convergence holds true in the $L^{2}$ sense. From (5.7) in Proposition 5.2 and (8.1) in Proposition 8.1, we get that $u \in L^{\frac{2(n+4)}{n}}\left(\mathbb{R}_{+} \times \mathbb{R}^{n}\right)$. An application of the Strichartz estimates (3.10) shows that the right hand side in (9.4) is like $o(1)$ in $L^{2}$ when $t_{0}$ and $t_{1}$ tend to infinity. In particular the convergence holds true in the $L^{2}$ sense. Now we prove (9.2). The first equation in (9.2) is a direct consequence of conservation of mass and of the convergence in $L^{2}$. Concerning the second equation, since $u \in Z\left(\mathbb{R}_{+}\right)$, we can find a sequence $t_{k} \rightarrow+\infty$ such that the $L^{\frac{2(n+4)}{n-4}}$-norm of $u\left(t_{k}\right)$ tends to zero. Combining this with conservation of mass, we get that the $L^{2^{\sharp}}$-norm of $u\left(t_{k}\right)$ tends to zero as $k$ tends to infinity. Let $\omega(t)=e^{i t\left(\Delta^{2}+\varepsilon \Delta\right)} u^{+}$. Then, we have

$$
\begin{aligned}
2 E\left(u_{0}\right) & =2 E\left(u\left(t_{k}\right)\right) \\
& =\left\|u\left(t_{k}\right)\right\|_{\dot{H}^{2}}^{2}-\varepsilon\left\|u\left(t_{k}\right)\right\|_{\dot{H}^{1}}^{2}+o(1) \\
& =\left\|\omega\left(t_{k}\right)\right\|_{\dot{H}^{2}}^{2}-\varepsilon\left\|\omega\left(t_{k}\right)\right\|_{\dot{H}^{1}}^{2}+o(1) \\
& =\left\|u^{+}\right\|_{\dot{H}^{2}}^{2}-\varepsilon\left\|u^{+}\right\|_{\dot{H}^{1}}^{2}+o(1),
\end{aligned}
$$

and letting $k \rightarrow+\infty$ we get that the second equation in (9.2) holds true. The continuity in $\dot{H}^{2}$ of the mapping $u_{0} \mapsto u^{+}$follows from estimate (3.20), Proposition 6.1 , and equation (9.5). The continuity in $L^{2}$ follows from the Strichartz estimates (3.10) and the a priori bound on the $Z$-norm in Proposition 8.1. We may proceed as when proving the control of the $L^{\frac{2(n+4)}{n}}\left(L^{\frac{2(n+4)}{n}}\right)$-norm in Proposition 5.2.

Conversely to Proposition 9.1 , it is easy to show that the operators $S_{ \pm}$are surjective.

Proposition 9.2. Let $n \geq 5$. For any $u^{+} \in H_{\text {rad }}^{2}$, respectively $u^{-} \in H_{\text {rad }}^{2}$, there exists a unique $u \in C\left(\mathbb{R}, H_{\text {rad }}^{2}\right)$, solution of (1.2) with $\lambda>0$ and $p=2^{\sharp}-1$, such that (9.1) holds true. In particular, $S_{ \pm}$are homeomorphisms from $H_{\text {rad }}^{2}$ onto $H_{\text {rad }}^{2}$. 
Proof. Again, by time reversal symmetry, we need only prove Proposition 9.2 for $u^{+}$. Let $\omega(t)=e^{i t\left(\Delta^{2}+\varepsilon \Delta\right)} u^{+}$. Then by the Strichartz estimates (3.10), $\omega \in W(\mathbb{R})$ and, given $\delta>0$, there exists $T_{\delta}$ such that the $W\left(\left[T_{\delta},+\infty\right)\right)$-norm of $\omega$ is less than $\delta$. For $u \in W\left(\left[T_{\delta},+\infty\right)\right)$, we define

$$
\Phi(u)(t)=\omega(t)-i \lambda \int_{t}^{\infty} e^{i(t-s)\left(\Delta^{2}+\varepsilon \Delta\right)}|u(s)|^{\frac{8}{n-4}} u(s) d s .
$$

It is easily seen that for $\delta$ sufficiently small, $\Phi$ defines a contraction mapping on the set

$$
\begin{aligned}
X_{T_{\delta}}= & \left\{u \in W\left(\left[T_{\delta},+\infty\right)\right) \cap L^{\frac{2(n+4)}{n}}\left(\left[T_{\delta},+\infty\right), L^{\frac{2(n+4)}{n}}\right) ;\|u\|_{W\left(\left[T_{\delta}, \infty\right)\right) \leq C \delta,} \leq\right. \\
& \left.\|u\|_{L^{\frac{2(n+4)}{n}}\left(\left[T_{\delta},+\infty\right), L^{\frac{2(n+4)}{n}}\right)} \leq C\left\|u^{+}\right\|_{L^{2}}\right\},
\end{aligned}
$$

equipped with the $L^{\frac{2(n+4)}{n}}\left(\left[T_{\delta},+\infty\right), L^{\frac{2(n+4)}{n}}\right)$-norm. Thus $\Phi$ admits a unique fixed point $u$. It follows from the Strichartz estimates (3.19) and (3.20) that $u \in$ $C\left(\left[T_{\delta},+\infty\right), H^{2}\right) \cap M\left(\left[T_{\delta},+\infty\right)\right)$. Besides, we can observe that

$$
u\left(T_{\delta}+t\right)=e^{i t\left(\Delta^{2}+\varepsilon \Delta\right)} u\left(T_{\delta}\right)+i \lambda \int_{T_{\delta}}^{T_{\delta}+t} e^{i(t-s)\left(\Delta^{2}+\varepsilon \Delta\right)}|u(s)|^{\frac{8}{n-4}} u(s) d s .
$$

Then, $u$ solves $(1.2)$ with $p=2^{\sharp}-1$ on $\left[T_{\delta},+\infty\right)$. Hence, using the radial symmetry assumption, we see from Theorem 1.1 that $u$ can be extended on $\mathbb{R}$. Now, (9.1) follows from (9.6) and the boundedness of $u$ in $W$ and $L^{\frac{2(n+4)}{n}}\left(L^{\frac{2(n+4)}{n}}\right)$-norms. Uniqueness follows from the fact that any radially symmetrical solution of (1.2) with $p=2^{\sharp}-1$ and $\lambda>0$ has a restriction in $X_{T}$ for some $T \geq T_{\delta}$, and uniqueness of the fixed point of $\Phi$ in such spaces. The continuity statements can be proved with similar arguments to those used in the proof of Proposition 9.1.

ACKNOWLEDGEMENT: The author expresses his deep thanks to Emmanuel Hebey for his constant support and for stimulating discussions during the preparation of this work. The author also expresses his deep thanks to the referee for very helpful comments that helped improve the manuscript.

\section{References}

[1] Ben-Artzi, M. Koch, H., and Saut, J.C., Disperion estimates for fourth order Schrödinger equations, C.R.A.S., 330, Série 1, (2000), 87-92.

[2] Bourgain, J., Global well-posedness of defocusing 3D critical NLS in the radial case, JAMS, 12 (1999), 145-171.

[3] Cazenave, T., Semilinear Schrödinger equations, Courant Lecture Notes in Mathematics, 10, New York University, Courant Institute of Mathematical Sciences, New York; American Mathematical Society, Providence, RI, (2003).

[4] Colliander, J., Keel, M., Staffilani,G., Takaoka, H., and Tao,T., Global well-posedness and scattering in the energy space for the critical nonlinear Schrödinger equation in $\mathbb{R}^{3}$. Ann. of Math. to appear.

[5] Fibich, G., Ilan, B. and Papanicolaou, G., Self-focusing with fourth order dispersion. SIAM J. Appl. Math. 62, No 4, (2002), 1437-1462.

[6] Fibich, G., Ilan, B. and Schochet, S. Critical exponent and collapse of nonlinear Schrödinger equations with anisotropic fourth-order dispersion. Nonlinearity 16 (2003), 1809-1821.

[7] Foschi, D., Inhomogeneous Strichartz Estimates, J. Hyp. Diff. Equ. 2 (2005), 1-24.

[8] Grillakis, M., On nonlinear Schrödinger equations, Comm. Part. Diff. Equations 25 (2000), $1827-1844$.

[9] Guo, B. and Wang, B. The global Cauchy problem and scattering of solutions for nonlinear Schrödinger equations in $H^{s}$, Diff. Int. Equ. 15 no 9 (2002), 1073-1083. 
[10] Hao, C. Hsiao, L. and Wang, B. Well-posedness for the fourth-order Schrödinger equations, J. of Math. Anal. and Appl. 320 (2006), 246-265.

[11] Hao, C. Hsiao, L. and Wang, B. Well-posedness of the Cauchy problem for the fourth-order Schrödinger equations in high dimensions, J. of Math. Anal. and Appl. 328 (2007) 58-83.

[12] Huo, Z. and Jia, Y. The Cauchy problem for the fourth-order nonlinear Schrödinger equation related to the vortex filament, J. Diff. Equ. 214 (2005), 1-35.

[13] Karpman, V.I., Stabilization of soliton instabilities by higher-order dispersion: fourth order nonlinear Schrödinger-type equations. Phys. Rev. E 53, 2 (1996), 1336-1339.

[14] Karpman, V.I., and Shagalov, A.G., Stability of soliton described by nonlinear Schrödingertype equations with higher-order dispersion, Phys D. 144 (2000) 194-210.

[15] Kato, T. On nonlinear Schrödinger equations, II. $H^{s}$-solutions and unconditional wellposedness. J. Anal. Math. 67 (1995), 281-306.

[16] Keel, M., and Tao, T. Endpoint Strichartz inequalities. Amer. J. Math. 120 (1998), 955-980.

[17] Kenig, C. and Merle, F., Global well-posedness, scattering, and blow-up for the energy-critical focusing nonlinear Schrödinger equation in the radial case, Invent. Math. 166 no 3 (2006), 645-675.

[18] Killip, R., Visan, M. and Zhang X., Energy-critical NLS with quadratic potentials preprint, (2006).

[19] Lin, J.E., and Strauss, W.A., Decay and scattering of solutions of a nonlinear Schrödinger equation. J. Funct. Anal. 30, (1978), 245-263.

[20] Levandosky, S. and Strauss, W., Time decay for the nonlinear beam equation. Meth. and Appl. of Anal. Vol 7 no 3 (2000), 479-488.

[21] Payne, L.E. and Sattinger, D.H., Saddle points and instability of nonlinear hyperbolic equations, Israel J. Math., 22, 1975, 273-303.

[22] Ryckman, E. and Visan, M., Global well-posedness and scattering for the defocusing energycritical nonlinear Schrodinger equation in $\mathbb{R}^{1+4}$, Amer. J. Math. 129 (2007), 1-60.

[23] Segata, J., Well-posedness for the fourth-order nonlinear Schrödinger type equation related to the vortex filament, Diff. Int. Equ. 16 no 7 (2003), 841-864.

[24] Segata, J., Remark on well-posedness for the fourth order nonlinear Schrödinger type equation, Proc. Amer. Math. Soc. 132 (2004), 3559-3568.

[25] Segata, J., Modified wave operators for the fourth-order non-linear Schrdinger-type equation with cubic non-linearity Math. Meth. in the Appl. Sci. 26 n 15 (2006) 1785-1800.

[26] Tao, T., Global well-posedness and scattering for the higher-dimensional energy-critical nonlinear Schrödinger equation for radial data, New York Journal of Math. 11 (2005), 57-80.

[27] Tao, T., Nonlinear dispersive equations, local and global analysis. CBMS. Regional Conference Series in Mathematics, 106. Published for the Conference Boardof the Mathematical Science, Washington, DC; by the American Mathematical Society, Providence, RI, 2006. ISBN: 0-8218-4143-2.

[28] Tao, T. and Visan, M., Stability of energy-critical nonlinear Schrödinger equations in high dimensions, Electron. J. Diff. Eq. 118 (2005), 1-28.

[29] Visan, M., The defocusing energy-critical nonlinear Schrodinger equation in higher dimensions Duke Math J. to appear.

[30] Wang, B. Nonlinear scattering theory for a class of wave equations in $H^{s}$. J. Math. Anal. Appl. 296 (2004), 74-96.

Université de Cergy-Pontoise, Département de Mathématiques, Site de Saint-Martin,

2 avenue Adolphe Chauvin, 95302 Cergy-Pontoise cedex, France

E-mail address: Benoit.Pausader@math.u-cergy.fr 